\title{
Review of Second Harmonic Generation Measurement Techniques for Material State Determination in Metals
}

\author{
K. H. Matlack · J.-Y. Kim ・ L. J. Jacobs · J. Qu
}

Received: 11 July 2014 / Accepted: 12 November 2014 / Published online: 25 November 2014

(C) Springer Science+Business Media New York 2014

\begin{abstract}
This paper presents a comprehensive review of the current state of knowledge of second harmonic generation (SHG) measurements, a subset of nonlinear ultrasonic nondestructive evaluation techniques. These SHG techniques exploit the material nonlinearity of metals in order to measure the acoustic nonlinearity parameter, $\beta$. In these measurements, a second harmonic wave is generated from a propagating monochromatic elastic wave, due to the anharmonicity of the crystal lattice, as well as the presence of microstructural features such as dislocations and precipitates. This article provides a summary of models that relate the different microstructural contributions to $\beta$, and provides details of the different SHG measurement and analysis techniques available, focusing on longitudinal and Rayleigh wave methods. The main focus of this paper is a critical review of the literature that utilizes these SHG methods for the nondestructive evaluation of plasticity, fatigue, thermal aging, creep, and radiation damage in metals.
\end{abstract}

Keywords Second harmonic generation - Microstructural evolution in metal $\cdot$ Nonlinear ultrasonic measurements

K. H. Matlack (凶)

Department of Mechanical and Process Engineering, Swiss Federal Institute of Technology (ETH Zurich), Zurich, Switzerland e-mail: matlackk@ethz.ch

J.-Y. Kim · L. J. Jacobs

School of Civil and Environmental Engineering,

Georgia Institute of Technology, Atlanta, GA, USA

J. Qu

Department of Civil and Environmental Engineering,

Northwestern University, Evanston, IL, USA

\section{Introduction}

Nonlinear ultrasonic methods have the powerful ability to characterize microstructural features in materials. Compared to more conventional linear ultrasonic methods that can detect cracks or features on the order of the wavelength of the ultrasonic wave, nonlinear methods are sensitive to microstructural features that are orders of magnitude smaller than the wavelength. Second harmonic generation (SHG) is a type of nonlinear ultrasonic method that has been shown to be capable of detecting and monitoring microstructural changes in metals. The physical mechanism of this is as follows: as a sinusoidal ultrasonic wave propagates through a material, the interaction of this wave with microstructural features generates a second harmonic wave. This effect is quantified with the measured acoustic nonlinearity parameter, $\beta$. SHG measurement methods have received significant focus and attention in the literature in recent decades, as the reliability and integrity of structural components becomes increasingly important to ensure safe operation of critical structures in, for example, the energy, transportation, and aviation industry. This paper presents a review of SHG measurements and their applications, to provide a comprehensive summary of these techniques, to thoroughly explain these measurements to new comers in this field of research, and to show where advances in this field of research are needed.

SHG methods were first reported on back in the 1960s, with a series of papers by Breazeale et al. [1,2], work by Gedroitz and Krasilnikov [3], and another series by Hikata et al. $[4,5]$. Some of the first reported SHG measurements of anharmonicity were conducted by both Gedroitz and Krasilnikov [3], as well as Breazeale and Thompson [1]. In the latter, the second harmonic wave was measured in polycrystalline aluminum over increasing source voltage of the fundamental wave with quartz crystal transducers, showing the 
linear dependence of the generated second harmonic wave on the squared amplitude of the fundamental wave. Other initial experimental studies primarily focused on single crystals (e.g. copper [2,6-10], germanium [11], aluminum [5,12]), and on fused silica [12-15].

There are a multitude of other nonlinear ultrasonic NDE techniques. Acousto-elasticity measurements are based on the phenomenon that changes in the stress state of a nonlinear medium cause a change in the wave velocity [16], and measurements exploiting the acousto-elastic effect have shown the sensitivity of material nonlinearity to fatigue microcracks [17]. Nonlinear elastic wave spectroscopy (NEWS) methods-such as nonlinear resonant ultrasound spectroscopy (NRUS) and nonlinear wave modulation spectroscopy (NWMS) techniques-have been extensively used to characterize a variety of materials such as geomaterials, rock, and concrete [18]. These techniques exploit the nonlinear hysteretic nature of these materials. NRUS techniques look at a resonance frequency shift with increasing amplitude excitation [18-20]. NWMS techniques typically look at modulation frequencies as a result of mixing a very low frequency (sometimes induced by a shaker or mechanical tapping) and a high frequency ultrasonic wave $[21,22]$. Nonlinear time reversal methods have been used in geophysics applications and detecting surface defects $[23,24]$. Nonlinear mixing techniques [25,26] utilize two input waves at different ultrasonic frequencies, and have recently gained attention in the literature. Material nonlinearity causes the generation of sum or difference frequencies, depending on input wave polarities, where the two waves interact. Nonlinear mixing techniques have the potential to be isolated from equipment and system nonlinearities inherent in harmonic generation experiments.

Second harmonic generation measurements have shown to be very applicable in detecting microstructural changes in metals prior to macroscopic damage and/or microcracking. Some experimental techniques have the unique advantage of simplicity in utilizing commercial transducers and standard ultrasonic testing equipment. Much of the current work on SHG techniques is focused on how these measurements can be applied to interrogate real materials under realistic loading conditions. A variety of different measurement techniques have been developed to interrogate different geometries and damage types. This review is intended to extend previous reviews [27,28], and will specifically focus on second harmonic generation experiments and applications explored particularly within the recent years. This review begins with a theoretical overview of SHG of longitudinal and Rayleigh waves, followed by a review of different microstructural contributions to $\beta$. Then, the different SHG measurement techniques reported throughout the literature are reviewed, followed by a discussion on how these measurements have been applied to monitor microstructural evolution during material damage. Finally, an outlook on potential future directions of SHG measurement research is given.

\section{Review of SHG Theory}

\subsection{Longitudinal Waves}

Consider longitudinal wave propagation through an isotropic medium with a quadratic nonlinearity, which results from a non-quadratic interatomic potential in crystalline materials. The equation of motion, simplified to one-dimension is:

$\rho \frac{\partial^{2} u}{\partial t^{2}}=\frac{\partial \sigma_{x x}}{\partial x}$

where $\rho$ is the material density, $u$ is the particle displacement, $\sigma_{x x}$ is the normal stress in the $x$-direction, $x$ is the material coordinate, and $t$ is time. The constitutive equation for a quadratic nonlinearity is given as:

$\sigma_{x x}=\sigma_{0}+E_{1}\left(\frac{\partial u}{\partial x}\right)+\frac{1}{2} E_{2}\left(\frac{\partial u}{\partial x}\right)^{2}+\ldots$

where $E_{1}$ and $E_{2}$ are the appropriate second- and third-order elastic constants. The nonlinear wave equation can thus be derived as

$$
\frac{\partial^{2} u}{\partial t^{2}}=c^{2}\left[1-\beta \frac{\partial u}{\partial x}\right] \frac{\partial^{2} u}{\partial x^{2}}
$$

where $\beta$ is the nonlinearity parameter, and $c$ is the longitudinal wave velocity in the material. For a material in its virgin state, $\beta$ is equivalent to the lattice anharmonicity component, $\beta_{0}$, and is a function of second- and third-order elastic constants of the material:

$\beta_{0}=-\left(\frac{3 C_{11}+C_{111}}{\sigma_{0}+C_{11}}\right)$

where $C_{11}$ and $C_{111}$ are the second- and third-order Brugger elastic constants, respectively, written in Voigt notation, and $\sigma_{0}$ is the initial stress in the material. Equation (4) assumes wave propagation in the (100) direction, and is also an exact solution for isotropic materials. Note that $\beta$ depends on the crystalline structure and symmetry of the material, which was shown in [29] through calculations of $\beta$ for pure mode propagation for various single-crystals.

The time harmonic solution to Eq. (3), assuming plane wave propagation, has the form:

$$
u=A_{1} \sin (\kappa x-\omega t)+\frac{\beta A_{1}^{2} x \kappa^{2}}{8} \cos (2 \kappa x-2 \omega t)+\ldots
$$


where $\omega(=2 \pi f)$ is the radial frequency of the wave at frequency $f, \kappa(=\omega / c)$ is the wavenumber of the propagating wave, $A_{1}$ is the amplitude of the first harmonic wave. The coefficient in front of the second term is $A_{2}$, the amplitude of the second harmonic wave, which assumes the absence of attenuation, diffraction, scattering, and assumes plane wave propagation. By simply rearranging this amplitude term, the nonlinearity parameter can be expressed in terms of acoustic quantities, i.e.:

$\beta=\frac{8 A_{2}}{A_{1}^{2} x \kappa^{2}}$

When written in this form, $\beta$ is generally referred to as the acoustic nonlinearity parameter. Thus by measuring the second harmonic wave amplitude, along with the first harmonic amplitude, wavenumber, and propagation distance, one can determine the acoustic nonlinearity parameter, $\beta$. This derivation can be expanded to three dimensions [30], and has been derived for Rayleigh waves [31] and explored for Lamb waves [32]. The same general form of $A_{2}$ has been shown for Rayleigh waves, in terms of dependence on propagation distance, wavenumber, and first harmonic wave. Experimental results have shown the same relationship of $A_{2}$ proportional to $A_{1}^{2}$ holds true for Lamb waves as well [82-85].

Note that the energy transfer from the first to second harmonic wave in SHG is very small compared to the energy of the propagating first harmonic wave, such that the decrease in $A_{1}$ due to the energy transfer is insignificant for small propagation distances. Further, in most experiments, the amplitude $A_{2}$ is orders of magnitude smaller than $A_{1}$. In the present considerations, we assume the wave propagation distance is small enough such that the energy loss of $A_{1}$ is negligible compared to the total energy of the propagating first harmonic wave.

For real materials and finite propagation distances, attenuation (dissipation, scattering, diffraction) will further decrease the amplitudes of the first and second harmonic waves with increasing propagation distance. The effect on the generated second harmonic wave when attenuation effects are non-negligible is derived elsewhere for longitudinal waves $[5,16,33]$. Attenuation effects are non-negligible at larger propagation distances, i.e. when $x\left(\alpha_{2}-2 \alpha_{1}\right)<<1$ does not hold, and the acoustic nonlinearity parameter for this case is given as $[5,16]$ :

$\beta_{\text {atten }}=\beta \frac{x\left(\alpha_{2}-2 \alpha_{1}\right)}{\left\{1-\exp \left[-\left(\alpha_{2}-2 \alpha_{1}\right) x\right]\right\}}$

where $\alpha_{1}$ is the attenuation coefficient at the first harmonic frequency, and $\alpha_{2}$ is the attenuation coefficient at the second harmonic frequency. Note that in Eq. (7) it is assumed that
SHG and attenuation effects occur independently, and in the limit $\alpha_{2} \rightarrow 2 \alpha_{1}$, the measured $\beta$ equals the actual $\beta$.

A more accurate expression for the acoustic nonlinearity parameter can be found by accounting for the on-axis diffraction effects of both the first and second harmonic wave. A diffraction correction $\left|D_{\beta}\right|$ to $\beta$ was introduced by Hurley et al. [14] as:

$\left|D_{\beta}\right|=\frac{|D(\omega)|^{2}}{|D(2 \omega)|}$

where $|D(\omega)|$ and $|D(2 \omega)|$ are the diffraction corrections to the first and second harmonic waves, respectively. The acoustic nonlinearity parameter scaled by this diffraction correction is thus $\beta_{D}=\beta D_{\beta}$.

The linear diffraction correction, i.e. the diffraction correction for the propagating first harmonic wave, has been derived in full previously [34] for a piston source such that the amplitude is constant across the source. This diffraction correction is given by:

$D(\omega, x, a)=1-\exp \left(-i \kappa a^{2} / x\right)\left[J_{0}\left(\kappa a^{2} / x\right)+i J_{1}\left(\kappa a^{2} / x\right)\right]$

where $a$ is the transducer radius, and $J_{0}$ and $J_{1}$ are Bessel functions of the first kind. In actuality, transducers are not a perfect piston source and there is some spatial distribution of amplitude over the surface of the transducer face, which could potentially approximate a Gaussian distribution [35].

The diffraction of the second harmonic wave is spatially different than that of the first harmonic. The wave is generated not by the transducer (in a perfect system at least), but by the propagating first harmonic wave, which is diffracting over propagation distance. This nonlinear diffraction can be physically interpreted as follows: at each instance that a portion of the second harmonic wave is generated, that portion will then diffract linearly over the remainder of the propagation distance to the receiving transducer. This nonlinear diffraction effect has been derived as $[14,36]$ :

$D(2 \omega, x, a)=\frac{\left|\int_{0}^{x}[D(\omega, x-\sigma / 2, a)]^{2} d \sigma\right|}{x}$

where $D(\omega, x, a)$ is the linear diffraction correction given in Eq. (9).

\subsection{Rayleigh Surface Waves}

Consider a Rayleigh surface wave propagating in the positive $x$ direction in an isotropic infinite half-space, with direction $z$ pointing into the half-space, with a weak quadratic nonlinearity. The Rayleigh wave motion along a stress-free surface, 
assuming plane wave propagation, can be decomposed in terms of its shear and longitudinal wave components, which for a propagating sinusoidal wave is given as:

$u_{x}=A_{1}\left(e^{-p z}-\frac{2 p s}{\kappa_{R}^{2}+s^{2}} e^{-s z}\right) e^{i\left(\kappa_{R} x-\omega t\right)}$

$u_{z}=i A_{1} \frac{p}{\kappa_{R}}\left(e^{-p z}-\frac{2 \kappa_{R}^{2}}{\kappa_{R}^{2}+s^{2}} e^{-s z}\right) e^{i\left(\kappa_{R} x-\omega t\right)}$

where $p^{2}=\kappa_{R}^{2}-\kappa_{p}^{2}, s^{2}=\kappa_{R}^{2}-\kappa_{s}^{2}$, and $\kappa_{R}, \kappa_{P}$, and $\kappa_{S}$ are the wavenumbers for the Rayleigh, longitudinal, and shear waves, respectively. The second harmonic wave displacement components can be approximated at a sufficiently far distance as $[37,38]$ :

$u_{x}(2 \omega)=A_{2}\left(e^{-2 p z}-\frac{2 p s}{\kappa_{R}^{2}+s^{2}} e^{-2 s z}\right) e^{i 2\left(\kappa_{R} x-\omega t\right)}$

For isotropic materials, the acoustic nonlinearity due to shear waves vanishes due to symmetry conditions, so the generated second harmonic wave is purely due to the longitudinal wave component of the Rayleigh wave. Herrmann et al. [38] derived the acoustic nonlinearity parameter in terms of out-of-plane components of the first and second harmonic amplitude as:

$\beta=\frac{\bar{u}_{z}(2 \omega)}{\bar{u}_{z}^{2}(\omega) x} \frac{i 8 p}{\kappa_{P}^{2} \kappa_{R}}\left(1-\frac{2 \kappa_{R}^{2}}{\kappa_{R}^{2}+s^{2}}\right)$

where the overbar indicates the displacement is evaluated at $z=0$.

As can be seen in Eq. (14), the dependence of $\beta$ on first and second harmonic amplitude, as well as on propagation distance is the same for nonlinear Rayleigh waves as it is for nonlinear longitudinal waves, at least under the plane wave assumption. The dependence on propagation distance is generally exploited in nonlinear Rayleigh wave measurementsthe wave propagation distance is varied over multiple measurements of first and second harmonic wave amplitude, and a relative measure of $\beta$ can thus be made.

Since nonlinear Rayleigh waves are typically used for longer propagation distances, accounting for attenuation [39] and diffraction effects $[35,39,40]$ are crucial for accurate measurements of $\beta$. Shull et al. [35] explored diffraction effects of nonlinear Rayleigh waves both theoretically and numerically for a general forcing function, a uniform line source, and a Gaussian source. For example, a line source with a Gaussian distribution along the $y$-axis is defined by:

$w(y)=v_{0} e^{-(y / a)}$

where $v_{0}$ is the source amplitude and $a$ is the half-length of the line source. The first harmonic wave velocity component

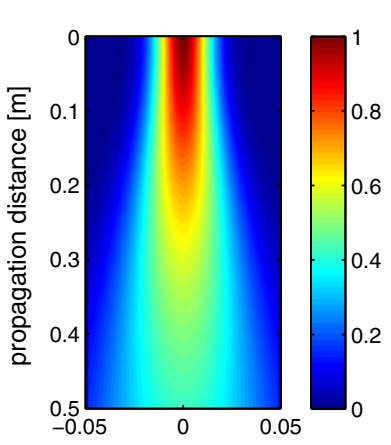

Sample/source width [m]

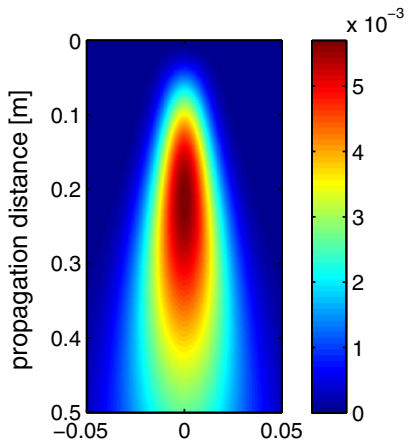

Sample/source width [m]
Fig. 1 Amplitude of first harmonic from Eq. (16) (left) and second harmonic from Eq. (17) (right) Rayleigh wave with propagation distance and sample width, including diffraction and attenuation effects, at $\mathrm{y}=0$ [40]. Results normalized by input first harmonic amplitude (reproduced from [40] with permission from Elsevier)

for this forcing function has been derived as [35]:

$v_{1}(x, y)=\frac{v_{0} e^{-\left(\alpha_{1} x\right)}}{\sqrt{1+i x / x_{0}}} \exp \left(-\frac{(y / a)^{2}}{1+i x / x_{0}}\right)$

where $x_{0}=\kappa_{R} a^{2} / 2$ is the Rayleigh distance that marks the transition from the near field to the far field diffraction region of the source, where $\kappa_{R}$ is the Rayleigh wavenumber. The second harmonic wave velocity component can be written as:

$v_{2}(x, y)=\beta_{11} v_{0}^{2} D_{R}(2 \omega)$

where $\beta_{11}$ is a nonlinearity parameter defined elsewhere [35,37], and $D_{R}(2 \omega)$ is the complex diffraction coefficient for the propagating and generated second harmonic Rayleigh wave, which is given in full in [35]. The first and second harmonic Rayleigh wave amplitude with propagation distance including diffraction and attenuation effects, i.e. Eqs. (16) and (17), are plotted in Fig. 1 [40]. These plots are normalized to the input first harmonic amplitude at $x=0$. In these plots, it is assumed that $\alpha_{n}=n^{4} \alpha_{1}$, and note that the propagation distance, $x$, is plotted on the vertical axis on these plots, and the sample/source width, $y$, is plotted on the horizontal axis.

The proportionality of the generated second harmonic wave remains the square of the fundamental at a given propagation distance $x$, i.e. $v_{2}(y) \propto v_{1}^{2}(y)$, though the dependence of $v_{2}(y)$ on propagation distance is obscured. While the linear dependence of $v_{2}(y)$ on propagation distance has been shown to be a good approximation for short propagation distances in many metals [38,41-43], the full solution incorporating attenuation, diffraction, and source effects will result in a more accurate determination of $\beta$. 


\subsection{Microstructural Contributions to $\beta$}

The parameter $\beta$ depends on the crystalline structure of the material, and also on localized strain present in the material. This strain arises from microstructural features such as dislocations and precipitates, and dislocation contributions to $\beta$ can greatly exceed that of the lattice anharmonicity. This section provides a comprehensive review of different microstructural contributions to the magnitude of the acoustic nonlinearity parameter. Theoretical derivations of effects of dislocation pinning $[4,16,44]$, dislocation dipoles [45,46], precipitate-pinned dislocations [16,47-49], and cracks [50-52] are reviewed.

\subsubsection{Dislocation Pinning: Hikata et al. Model}

The dislocation motion contribution to acoustic nonlinearity was first developed by Suzuki et al. [53] and expanded on by Hikata et al. [4], and this model has been used to interpret a multitude of experimental results of second harmonic generation. The model is based on the dislocation string vibration model of Granato and Lücke [54], and considers dislocation bowing as a line segment pinned between two points, a distance $2 L$ apart. These pinning points can be grain boundaries, other dislocations, or point defects in the material. Assume a small but non-zero longitudinal stress, $\sigma$, with shear component $\tau$ such that $\tau=R \sigma$ where $R$ is the resolving shear factor, is then applied to this dislocation segment such that it bows out between the two pinning points. An approximation of this geometry is depicted in Fig. 2, where the radius of curvature, $r$, of the bowed segment and angle $\theta$ are annotated. Note that this stress can be thought of as either an internal residual stress or externally applied stress, but it is small enough such that the dislocation segment does not break away from the pinning points. An ultrasonic stress wave sets the pinned dislocation segment into a vibrational motion. However, the dynamics of the dislocation motion is neglected by assuming that the mass density of the dislocation line is negligible. Further, the dislocation dynamics that may occur during

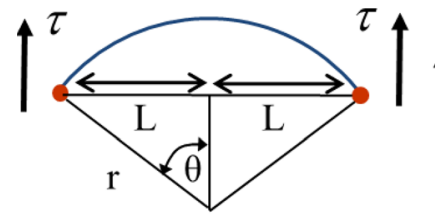

(a)

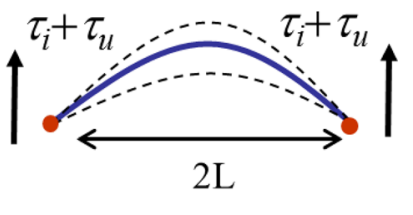

(b)
Fig. 2 a Diagram showing geometry of bowed dislocation segment of length $2 \mathrm{~L}$ between two pinning points and under an applied shear stress $\tau_{i}$, in terms of radius of curvature $\mathrm{r}$ and angle $\theta$. $\mathbf{b}$ Diagram showing movement of dislocation segment with superimposed ultrasonic stress on top of initial stress $\tau$ transient plastic deformation is not considered since this is not of the source of ultrasonic nonlinearity considered here.

Assuming the dislocation density is small enough such that bowed dislocations act independently of each other, the line tension, $T$, of this dislocation segment due to the applied stress was approximated as constant and independent of orientation as $T=\mu b^{2} / 2$, where $b$ is the Burgers vector and $\mu$ is the shear modulus. In the Hikata et al. model, the area swept out by the dislocation is approximated as a circular arc with a constant radius.

The total strain in the material can be written as a summation of the lattice strain plus the strain due to the dislocation motion, i.e. $\varepsilon=\varepsilon_{1}+\Omega \gamma$, where $\Omega$ is the conversion factor between shear and longitudinal strain and the shear strain $\gamma$ due to a distribution of bowed dislocations. Assuming the same form of the stress-strain relation as in Eq. (2) for the internal stress, the resulting stress-strain relationship due to the total strain in the material is:

$\varepsilon=\left(\frac{1}{E_{1}}+\frac{2 \Omega \Lambda L^{2} R}{3 \mu}\right) \sigma+\frac{E_{2}}{E_{1}^{3}} \sigma^{2}+\frac{4 \Omega \Lambda L^{4} R^{3}}{5 \mu^{3} b^{2}} \sigma^{3}$

with bowed dislocations density $\Lambda$, dislocation loop length $L$. Assuming a small ultrasonic stress $\Delta \sigma$ is superimposed on the internal stress $\sigma_{1}$, and assuming the lattice contribution of the nonlinearity parameter is $\beta_{0}=-E_{2} / E_{1}$, the change in nonlinearity parameter due to dislocation pinning can be written as:

$\Delta \beta_{p d}=\frac{24}{5} \frac{\Omega \Lambda L^{4} R^{3} C_{11}^{2}}{\mu^{3} b^{2}} \sigma_{1}$

Further details of this model can be found elsewhere $[4,16]$. Note that $E_{1}$ is defined as $\sigma_{0}+C_{11}$ in Eq. (4), but here it is assumed that the initial stress $\sigma_{0}$ is zero. It should be specifically noted that the internal stress $\sigma_{1}$ in this analysis, as well as the superimposed ultrasonic stress, is assumed to be much smaller than the yield stress of the material, such that dislocation displacement is small.

\subsubsection{Dislocation Pinning: Extensions of the Hikata et al. Model}

Later work by Cash and Cai [55] extended the Hikata et al. model to account for orientation-dependent line energy in the analytical model and verified with dislocation dynamics simulations. Results showed that the Hikata et al. model can accurately predict $\beta$ only for small values of Poisson's ratio for both screw and edge dislocations. However, at values of Poisson's ratio greater than about 0.2 (i.e. most metals), the dislocation dynamics simulations and developed analytical model show that relationship between $\beta$ and the applied stress is not in fact linear, and simulations show that $\beta$ can 
even be negative for an edge dislocation at small stresses. This result is not surprising since $\beta$ is known to be complex, but since current experimental techniques generally only measure amplitudes of the first and second harmonic waves, a negative $\beta$ would not be distinguishable from a positive $\beta$ of equal magnitude. However, if phase information were extracted from the experiments, a negative $\beta$ could be measured. This work provided sound evidence of the limitations of the widely utilized Hikata et al. model and called for further experiments to measure $\beta$ over increasing levels of applied stress to further explore this relationship.

Zhang et al. [56,57] extended the Hikata et al. model to account for the nonlinearity from screw and edge dislocations, however their work still considered the constant line tension model to express the area swept by the bowed dislocation segment as an approximation of a circular arc with constant radius. The model predicted that edge dislocations give rise to a larger acoustic nonlinearity than screw dislocations, and compared their predictions to previous SHG experiments on cold rolled 304 stainless steel [58]. While these SHG experimental results show better correlation with the Zhang models in [56,57] than the Cash-Cai model [55], significant variation from dislocation measurements taken from a previous paper were not included in the analysis. More importantly, the dislocation structures formed during cold rolling will likely have stronger effects on $\beta$ than the pure dislocation monopole contribution considered in these models.

Further work by Chen and Qu [59] extended the Hikata et al. model as well as the Cash-Cai model by developing a solution for pure and mixed dislocations with orientationdependent line energy in anisotropic crystals. They validate their model with molecular dynamic simulations. The contribution from pinned dislocations to $\beta$ from their results was derived as:

$\Delta \beta_{p d}=4 \Omega \Lambda L^{4} R^{2} b^{2}\left(\frac{C_{1111}}{C_{1212}}\right)^{2}\left(\frac{L}{b}\right)^{3} \bar{S}^{\prime \prime}\left(\tau_{n}\right)$

where $\bar{S}^{\prime \prime}\left(\tau_{n}\right)$ is the second derivative with respect to $\tau_{n}$ of the swept-out area of the dislocation segment under a shear stress, and $\tau_{n}$ is a dimensionless value proportional to the applied shear stress and the dislocation length. The authors present a procedure to determine $\bar{S}^{\prime \prime}\left(\tau_{n}\right)$ without needing to perform the numerical derivatives, and the reader is referred to the paper for more details [59]. Their model shows that the asymptotic solution derived by Cash and Cai [55] is a good approximation for the generated acoustic nonlinearity from screw dislocations, but does not accurately predict the full solution for $\beta$ due to edge dislocations. The authors show with a simulation experiment that their solution can track $\beta$ for changing dislocation lengths, but the crucial link to real SHG experiments has yet to be realized.

\subsubsection{Dislocation Dipoles}

Dislocation dipoles are formed when two dislocations of opposite sign move within some small distance $d$ of each other and become mutually trapped. The force-displacement relation of the dipole is a nonlinear relation, and as such it has been shown that when perturbed by an ultrasonic wave, this feature generates a nonzero component of acoustic nonlinearity $[16,45,46,60,61]$. The contribution to nonlinearity from dislocation dipoles, $\Delta \beta_{d p}$, has been derived as $[46,61]$ :

$\Delta \beta_{d p}=\frac{16 \pi^{2} \Omega R^{2} \Lambda_{d p} h^{3}(1-v)^{2} C_{11}^{2}}{\mu^{2} b}$

where $\Lambda_{d p}$ is the density of dislocation dipoles, and $h$ is the dipole height.

In fatigue damage, increased cyclic loading causes dislocation substructures to form. In wavy-slip metals (e.g. polycrystalline nickel [46], aluminum alloys [51], and copper single crystals [62] and references therein), these substructures initiate as vein structures that are regions of high dislocation density that ultimately becomes saturated with dislocation dipoles. Regions in between vein structures are referred to as channels, and generally have a few orders of magnitude less dislocation density. The vein structures can further transform into a stable persistent slip band structure (PSBs) that is a ladder-type configuration of vein regions. In planar slip metals, for example the IN100 nickel superalloy studied in [63] (and the references therein), the primary dislocation substructures are planar slip bands and intermittently activated persistent Luders bands (PLBs).

As an example, Cantrell has derived the total value for the nonlinearity parameter in wavy-slip metals for different contributions from dislocation monopoles, dipoles, and the different substructures as:

$\beta=\frac{\beta^{e}+f^{m p} \beta^{m p}+f^{d p} \beta^{d p}}{\left[1+f^{m p} \Gamma^{m p}+f^{d p} \Gamma^{d p}\right]^{2}}$

where $f^{m p}$ and $f^{d p}$ are the total volume fractions of dislocation monopoles and dipoles respectively, and $f^{m p} \beta^{m p}$ and the corresponding dipole term depend on specific $\beta$ and $f$ parameters in the veins, channels, and PSBs. The gamma factors depend on dislocation and material parameters and are given in full in [46].

\subsubsection{Precipitates}

Now consider the effect of a distribution of precipitates on the magnitude of $\beta$. Precipitates themselves do not have a significant effect on $\beta$ [47], but their interaction with dislocations has shown to give rise to a significant change in the 
Fig. 3 a Diagram of precipitate with radius $r_{p}$ embedded in a matrix with natural radius of $r_{a}$, and $\mathbf{b}$ diagram of a dislocation bending around a distribution of precipitates, with spacing of $\mathrm{L}$ (based on [66])

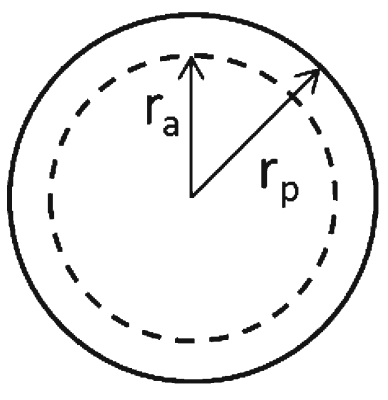

(a)

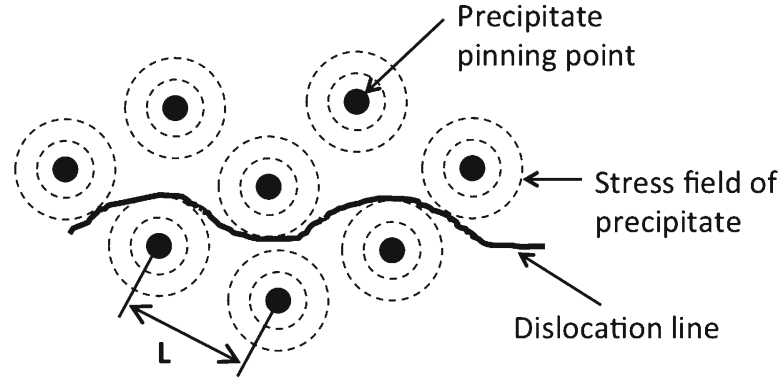

(b) magnitude of $\beta$ [47-49]. Effectively, the precipitate embedded in a surrounding matrix creates a local stress field which is then used as the applied stress on a pinned dislocation segment, as given in Eq. (19). Consider a precipitate with radius $r_{p}$ embedded in the matrix, with a precipitate-matrix lattice misfit parameter $\delta$ such that $r_{p}=r_{a}(1+\delta)$, where $r_{a}$ is the natural radius in the matrix, as depicted in Fig. 3a [64]. The precipitate is approximated as spherical and embedded in an isotropic medium, exerting a non-zero internal pressure $p_{0}$ on the matrix [46]. The stress, $\sigma_{r r}(r)$, in the radial direction at radius $r>r_{a}$ for this scenario are given in Eringen [65], as:

$\sigma_{r r}(r)=-p_{0} \frac{r_{p}^{3}}{r^{3}}$

Assuming the precipitate and matrix have different elastic properties, the stress in the matrix at radius $r$ due to this embedded precipitate can then be written as $[49,67]$ :

$\sigma_{r r}(r)=-4 \mu \delta\left[\frac{3 B_{p}}{3 B_{p}+4 \mu}\right] \frac{r_{p}^{3}}{r^{3}}$

where $B_{p}$ is the bulk modulus of the precipitate.

Then assume there is a distribution of these spherical precipitates embedded in a microstructure with dislocations present. The precipitates exert a local stress field as described above in its vicinity. Since a dislocation line is assumed to follow a contour of minimum energy, it is assumed that two precipitates a distance $L / 2$ away from each dislocation segment act on the dislocation segment, and contributions from other nearby precipitates are negligible. This scenario is depicted in Fig. 3b. Thus, the stress is evaluated at $r=L / 2$ [47]. We can then write Eq. (19) in terms of precipitate parameters [49] to find the change in the acoustic nonlinearity parameter due to precipitate-pinned dislocations, $\Delta \beta_{p p d}$ :

$\Delta \beta_{p p d}=\frac{1536}{5} \frac{\Omega R^{3} C_{11}^{2}}{\mu^{3} b^{2}}\left[\frac{3 B_{p}}{3 B_{p}+4 \mu}\right]\left(\Lambda L \bar{r}_{p}^{3}|\delta|\right)$

where the terms in parentheses are parameters that will most likely evolve throughout initial stages of material damage such as fatigue, thermal aging, or radiation damage (i.e. the dislocation and precipitate parameters). The precipitate spacing has been estimated as $L \propto N_{p}^{-1 / 3}$, where $N_{p}$ is the number density of total precipitates. The number density of precipitates is related to the volume fraction of precipitates, $f_{p}$, as $f_{p}=N_{p} V_{p}$, where $V_{p}=4 \pi / 3 \overline{r^{3}}$ is the average volume of each precipitate. Thus, through a simple substitution, $\Delta \beta_{p p d}$ can be written in terms of number density or volume fraction of precipitates. Note that these results are the same as derived in [49], and based off of other previous work as well [46-48]. Further, it is useful to reiterate, in simpler terms, the dependence of $\Delta \beta$ on precipitate and dislocation parameters:

$$
\begin{aligned}
& \Delta \beta_{p p d} \propto \frac{\Lambda \bar{r}_{p}^{3}|\delta|}{N_{p}^{1 / 3}} \\
& \Delta \beta_{p p d} \propto \Lambda \bar{r}_{p}^{3}|\delta|\left(\frac{\overline{r_{p}^{3}}}{f_{p}}\right)^{1 / 3}
\end{aligned}
$$

\subsubsection{Microcracks}

Higher harmonic generation from crack contacting surfaces as a function of stress was studied by Hirose and Achenbach [68] with the boundary element method. Nazarov and Sutin later derived the nonlinearity parameter of elastic solids with uniformly distributed, randomly oriented penny shape cracks [52]. They assumed that the contact of asperities on the crack faces is elastic and the overall nonlinearity originates from the nonlinear stress-strain relationship of the asperity contact. Cantrell later applied this model to determine the contribution of nonlinearity from fatigue-induced cracks, depending on the fatigue process parameters and as a percent of fatigue life [51].

First consider the volume change of a single crack which is produced by the elastic deformation of the asperities in the crack surface and then evaluate the average strain of the whole solid body due to the individual volume changes. Assuming the deformation in every crack face is uniform, the applied longitudinal stress and the average strain due to the volume change is related by: 


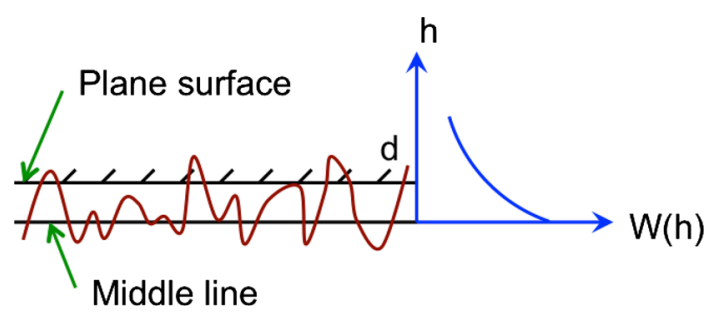

Fig. 4 Diagram of rough surface in contact with rigid plane [52]. Adapted with permission from Nazarov and Sutin [51]. Copyright 1997, Acoustical Society of America

$$
\begin{aligned}
\sigma & =E \alpha\left[\varepsilon-(\beta / 2) \varepsilon^{2}-(\delta / 6) \varepsilon^{3}\right] \\
\alpha & =\left(1+a N_{0} / 5\right)^{-1}, \beta=b N_{0} \alpha_{1}^{2} / 7, \\
\delta & =c N_{0} \alpha_{1}^{3}\left[1-27 \alpha_{1} b^{2} N_{0} / 49 c\right] / 9
\end{aligned}
$$

where $E$ is the Young's modulus, $N_{0}$ is the crack density (the number of cracks per unit volume), and $a, b, c$ are the coefficients in the Taylor expansion of the volume change in the normal stress $\left(\sigma_{n}\right.$, normal to the crack face). These expansion coefficients are determined from the analysis of the contact of multiple asperities in the crack. As shown in Fig. 4 [52], the contact of two rough crack surfaces is transformed to an equivalent problem of a rough surface in contact with a rigid flat surface [52]. Moreover, the asperity heights are assumed to follow an exponential distribution. Then, the normal contact stress resulting from asperities currently in contact with the rigid flat is written as follows:

$\sigma_{n}=-\int_{d}^{\infty} f(h-d) W(h) d h$

where $f$ is the force from the contact of a single spherical asperity on a rigid flat [69] and $W$ is the asperity distribution function which is given as $W(h)=n\left(\pi R^{2} h\right)^{-1}$ $\exp \left(-h / \sqrt{2} h_{0}\right)$, with $R$ being the radius of the spherical asperity and $h_{0}$ being the characteristic height of the rough surface. Note that Eq. (29) is the famous formula of Greenwood and Williamson [70]. The coefficients $a, b, c$ are obtained by evaluating Eq. (29) for the infinitesimal disturbance of ultrasound acting on the cracks as shown in [52]. It has been shown that the nonlinearity of contacting cracks is orders of magnitude higher than that of the dislocations substructures in cyclically fatigued metals [51]. Papers that include the effect of local plastic deformation [71] and adhesion of contacting asperities [72] have also appeared.

\section{Experimental Techniques}

Second harmonic generation measurements of the acoustic nonlinearity parameter can be conducted using multiple wave types, different generation and detection methods, and a variety of experimental set-ups. The general process is similar in all cases, where an ultrasonic tone burst at frequency $\omega$ is generated in the material, it propagates some distance through the material or structure, and the response is measured at some distance from the transmitter-specifically, the amplitudes of the first harmonic and second harmonic waves, $A_{1}$ and $A_{2}$, respectively, are extracted from the frequency response of the received signal. SHG measurements of $\beta$ have been realized using longitudinal waves e.g. [49,73-76], and Rayleigh surface waves e.g. [38,39,43,77-81]. Lamb wave (or plate) modes are also utilized for SHG measurements, e.g. [32,82-85], but this review will not discuss these techniques due to added complications with their dispersive and multimodal characteristics. This section gives a detailed overview of current experimental methods, measurement techniques, set-ups, and post-processing used for both longitudinal and Rayleigh wave SHG measurements of $\beta$.

\subsection{Longitudinal Waves}

Measurements of $\beta$ using longitudinal waves have been conducted with contact piezoelectric transducers [58,74$76,86,87]$, capacitive (electrostatic ultrasonic) transducers $[6,11,12,14,33,73,88,89]$, and laser interferometer detection $[14,49,90,91]$. An absolute measure of $\beta$ is possible with longitudinal waves using either capacitive transducers $[12,73]$ or contact piezoelectric transducers using a calibration procedure $[92,93]$ in which the absolute displacement amplitude of the first and second harmonic waves can be measured. As an example, absolute measurements of $\beta$ have been made extensively in fused silica materials, where values of $\beta$ range from 9.7 to 14 [12-15,90,92]. Absolute measurements have also been made in heat treated aluminum alloys where values of can range from 4 to 12 [92,94,95], as well as heat treated Inconel 718 with $\beta$ ranging from 3.5 to 7 and 7.5 to 9 depending on the specific heat [33]. Further, absolute measurements have been made most recently in fatigued single crystal copper, with values of $\beta$ over fatigue damage ranging from 7.8 to 13.2 and 10.3 to 21.3 depending on orientation [62].

Throughout the recent literature, $\beta$ has more typically been measured as a relative parameter since it is more practical in an in-situ setting to measure the relative amplitudes of $A_{1}$ and $A_{2}$ rather than the absolute physical displacement of the first and second harmonic waves. In the simplification of a relative measure of nonlinearity, the voltage amplitudes of the first and second harmonic are instead measured and the relative acoustic nonlinearity parameter is calculated, which is defined as:

$\beta^{\prime}=\frac{A_{2}^{v}}{\left(A_{1}^{v}\right)^{2}} \frac{8}{x \kappa^{2}}$ 
where recall that $\kappa$ is the wavenumber as defined in Eq. (5).

Note that the change in the relative acoustic nonlinearity parameter is proportional to the change in the absolute acoustic nonlinearity parameter and thus the material property and microstructural changes it encompasses, with respect to its initial state. A measurement of $\beta^{\prime}$ can be made by varying the input amplitude and measuring the resulting first and second harmonic amplitudes, and then calculating the slope of the linear fit between $\left(A_{1}^{v}\right)^{2}$ and $A_{2}^{v}$. While the amplifier, transducers, and/or coupling may excite a nonlinearity (referred to as the system nonlinearity) that is also proportional to the first harmonic squared, these measurements can be used to detect changes in material nonlinearity, since the system nonlinearity will remain constant over all the measurements. Note that in measurements utilizing longitudinal waves, the propagation distance and the wavenumber should be kept constant, otherwise $\beta^{\prime}$ must be scaled accordingly.

\subsubsection{Piezoelectric Methods: Relative Amplitude Measurements}

A longitudinal wave relative measurement of $\beta$ (meaning a measure of only the voltage amplitudes of $A_{1}$ and $A_{2}$ ) has been widely utilized throughout the recent literature [49,58,74-76,87,96-101]. Contact piezoelectric transducers offer a robust solution since these transducers are typically used in other linear ultrasonic measurements, sample surface preparation is not extreme (typically involving hand polishing of the contact surfaces), and can be purchased offthe-shelf for relatively low cost. However, transducers must be bonded (or coupled using a liquid coupling agent) to the sample surface which can introduce measurement variation if conditions are not accounted for or not kept consistent [96].

These measurements are made by fixing a transmitting and receiving transducer on opposite sides of the sample, using some acoustic coupling between the transducer face and the material (e.g. light oil, ultrasonic coupling gel, phenyl salicylate, or epoxy). Only a thin layer of coupling is needed, and care should be taken to ensure no air bubbles contaminate the coupling area. It has generally been found that a light oil coupling works better than thicker ultrasonic coupling gels, and much better than most adhesives due to inherent inconsistencies in bond quality after solidification. A fixture that can apply a consistent force on the transducer is crucial to measurement repeatability, since this directly influences the coupling condition between the transducer and sample. Liu et al. [102] showed that a low-level coupling force (2$5 \mathrm{~N}$ ) produced most consistent SHG results. A technique to minimize coupling effects on the nonlinearity measurement has been proposed in [96], in which the authors report a decrease in measurement variation by half using light oil coupling.
Transducers must be accurately aligned to each other, particularly since wavelengths in metal materials for relevant frequencies will likely be less than $1 \mathrm{~mm}$, so slight misalignments would result in smaller than accurate received amplitudes. In general, excitation frequencies in the range of $1-5$ $\mathrm{MHz}$ have been most utilized throughout the literature. The transmitting transducer has a center frequency at approximately the first harmonic frequency, and the receiving transducer has a center frequency at approximately the second harmonic frequency. While this receiving transducer will simultaneously detect the first and second harmonic amplitude, tuning it to the second harmonic frequency is crucial since that amplitude will be a few orders of magnitude smaller than the first harmonic wave amplitude. It has also been proposed to select the operating first harmonic frequency based on the transmitting transducer frequency response, such that the response of the transmitting transducer at the second harmonic frequency is minimized [102].

Common piezoelectric materials used as contact transducers for SHG measurements are lead-zirconium-titanate (PZT) and lithium niobate. PZT material is a ceramic that has a high efficiency yet is highly nonlinear [103]. So, this material will introduce system nonlinearity into the experiments. Lithium niobate on the other hand is not as efficient as PZT, but the material has a much smaller nonlinearity than PZT.

A linear amplifier, such as a RITEC amplifier, and a function generator is used to excite a sinusoidal wave in the transmitting transducer. Note that the RITEC amplifier has built-in frequency filtering capabilities that can accurately detect harmonic amplitudes. The amplifier should be inherently linear, and have the capability of exciting a high-powered ultrasonic wave, for example $1,200 \mathrm{~V}_{p p}$ on a $50 \Omega$ load. As many cycles as possible should be used to excite as much acoustic energy in the sample as possible to accurately detect the second harmonic wave amplitude above the signal noise level. Too many cycles such that successive reflections between sample boundaries interfere with the received signal should be avoided, particularly if small deviations in sample thicknesses (e.g. slight differences in thicknesses among different samples, or deviation in parallelism of the sample faces) are expected that would change how the reflected signal would interfere with the incoming wave. An oscilloscope or data acquisition system is used to receive the time domain signal, which is generally an average of multiple signals to improve the signal to noise ratio (256-512 averages have been reported as sufficient in some current experimental setups). Then the fast Fourier transform (FFT) is calculated to determine the amplitude of the signal as a function of frequency, in order to extract the relative amplitudes of the first and second harmonic waves. The pulse inversion technique, which can suppress the fundamental and odd harmonics, has also been utilized to more accurately extract the small second harmonic wave [74]. This process is repeated for different 
input amplitudes, and then the entire measurement procedure is repeated multiple times to achieve statistically significant data.

The exact values of input amplitude for these experiments can be crucial. Amplifiers must excite a stress wave with amplitude above the so-called "Buck hook" region, where $\beta$ is known to be largely dependent on amplitude. In this region, $\beta$ increases to a maximum and drops off rapidly with excitation voltage [89,104-106]. A damped oscillatory behavior of $\beta$ has been shown in regions above the Buck hook, which is a result of the Peierls-Nabarro barrier stress associated with dislocation motion $[89,105]$, but this oscillatory behavior is greatly damped in polycrystalline metals due to the random orientations of slip systems (and thus random values of the Schmid factor) [89]. As an example, the Buck hook region in Al2024 has been shown to be about $0.15-1.5 \mathrm{MPa}$, and about 0.1-1.3 MPa in IN100 [89]. Above this stress amplitude, $\beta$ has shown to be relatively constant, unless extremely high stress amplitudes are excited such that dislocations break away from pinning points. Therefore, the oscillatory behavior of $\beta$ on stress amplitude in non-polycrystalline materials must be considered in second harmonic generation measurements.

\subsubsection{Piezoelectric Methods: Absolute Amplitude Measurements}

Absolute measurements of $\beta$, through measuring the absolute displacement of the fundamental and second harmonic amplitudes of the received wave, are possible with piezoelectric contact transducers through a reciprocity-based calibration procedure. This method was originally formulated by Dace et al. $[92,93]$ and utilized in subsequent works, e.g. $[14,33,74]$. The purpose of the calibration procedure is to calculate the frequency-dependent transfer function, $H(\omega)$, of the receiving transducer by measuring the voltage and current signals of an echo from the stress-free back wall of the specimen. Then, a measured current signal can be directly converted to the displacement in the following way:

$A(\omega)=H(\omega) I^{\prime}(\omega)$

where $A(\omega)$ is the displacement amplitude and $I^{\prime}(\omega)$ is the current signal measured during the nonlinear measurement. The transfer function is defined as:

$H(\omega)=\sqrt{\frac{I_{\text {in }}(\omega) V_{\text {out }}(\omega) / I_{\text {out }}(\omega)+V_{\text {in }}(\omega)}{2 \omega^{2} \rho c_{L} a I_{\text {out }}(\omega)}}$

where $I_{\text {in }}, I_{\text {out }}, V_{\text {in }}$, and $V_{\text {out }}$ are the input and output current and voltage signals measured in the calibration measurement, $\rho$ is the material density, $c_{L}$ is the longitudinal wave speed, and $a$ is the transducer radius.
The experimental procedure is as follows. The receiving transducer is mounted on the sample, with a small amount of liquid coupling or a solid coupling such as phenyl salicylate. The receiving transducer must be attached to the sample such that when the transmitting transducer is later attached to the sample, the contact conditions of the receiving transducer are not disturbed, since this will change the transfer function. The receiving transducer is used in pulse-echo mode and excited with a broadband signal. A voltage and current probe are used to measure the input voltage and current to the transducer, as well as the output voltage and current signals of the reflected signal. The calibration procedure is based off of linear electroacoustic reciprocity, such that the reflection of the pulse is from a stress-free surface, so the transmitting transducer must not be attached to the sample surface during the calibration. After the calibration, the transmitting transducer is attached to the opposite side of the sample and aligned with the receiving transducer. The transmitting transducer is excited with a tone burst signal at the fundamental frequency, and the receiving transducer is used to measure the output current $I^{\prime}(\omega)$. The experimental setup used in Barnard et al. [33] for these absolute $\beta$ measurements is shown in Fig. 5.

\subsubsection{Capacitive Methods}

Absolute measurements of $\beta$ first became possible through the development of the capacitive receiver for SHG measurements by Gauster and Breazeale [11]. In this method, which is extensively described in [88,107], a broadband airgap capacitive transducer is held at a small distance above the sample $(1-10 \mu \mathrm{m})$. A gated tone burst sinusoidal signal is generated with a transmitting transducer bonded to one side of the sample. The wave propagates through sample, and the capacitive transducer is held a small distance (about 1-10 $\mu \mathrm{m}$ ) away from the opposite side of the sample. The sample (or a conductive coating on the sample surface) acts as one plate of a capacitor, and the impinging ultrasonic wave causes the gap spacing between the sample and the transducer to vary with time. In the recent literature, these methods have been extensively and very successfully used by Cantrell and Yost and co-authors, e.g. [45,62,89]. This detection system has a reported sensitivity of $10^{-16} \mathrm{~m}$, which is more than sufficient to detect the displacement amplitudes of the propagated first and second harmonic wave [108]. The first and second harmonic displacement amplitudes, $A(\omega)$, are determined from:

$A(\omega)=\frac{d V_{0}(\omega)}{2 V_{b}}$

where $d$ is the gap spacing, $V_{0}$ is the output voltage, and $V_{b}$ is the applied bias voltage [107]. $V_{0}$ is determined as the measured voltage at the oscilloscope, using a narrow band pass filter at $\omega_{1}$ and $\omega_{2}$. Note that the factor of 2 in the denominator of Eq. (34) is to account for the doubling of the displacement 
Fig. 5 Experimental schematic for SHG calibration and absolute measurement of $\beta$ using piezoelectric transducers (adapted from [33] with permission from Springer)

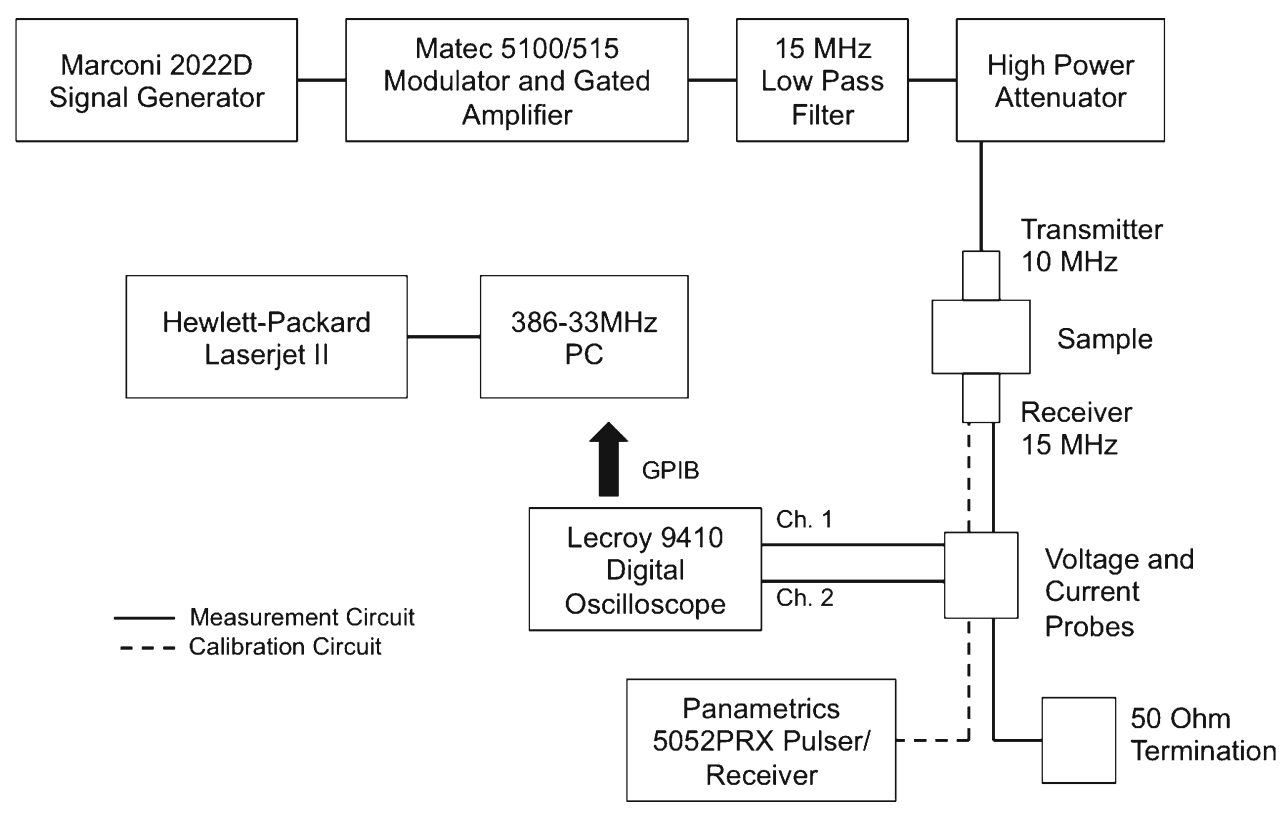

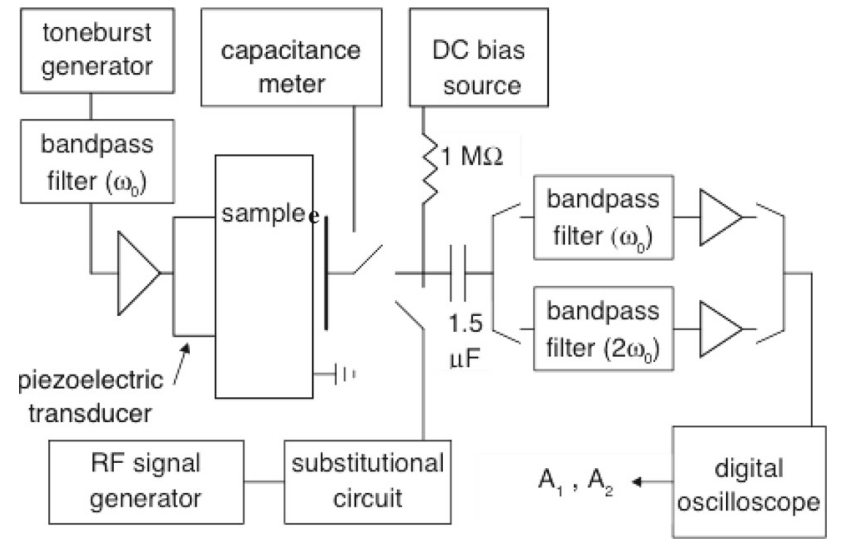

Fig. 6 Schematic of experimental setup for capacitive dection for SHG measurements. This is based off the experiments developed in [88,107]. (Reproduced from Hurley et al. [14] with permission from IOP Science)

amplitude when reflected from a free surface. To further calculate the output voltage from this measured voltage, the signal received on the oscilloscope is matched by generating an RF signal with a highly accurate function generator through the same narrow band pass filters and matching the amplitude of the measured voltage from the nonlinear measurement; the output voltage from the function generator is used as $V_{0}$ in the calculation of the displacement amplitude in Eq. (34). A schematic of an example experimental setup utilizing the capacitive detection system for SHG measurements is shown in Fig. 6.

While this method offers a direct way of measuring absolute displacements of the first and second harmonic waves compared to piezoelectric transducers (which require a series of calibrations for these absolute measurements [92]) sample preparation is cumbersome, requiring an optically flat and parallel sample surface over the receiver area and a small gap of only a few microns.

\subsubsection{Laser Methods}

Laser ultrasonic SHG methods have the unique advantage of being a noncontact measurement, with the capability of detecting an absolute, point-like displacement measurement. These methods have been recently reviewed in detail, in terms of guided wave NDE [109], and a review of non-contact ultrasonic measurements in general can be found in [110]. Absolute measurements of $\beta$ in fused silica have been made using a heterodyne [90] and homodyne [14] interferometer, and in both cases measurements of $\beta$ corresponded well with literature values. A heterodyne laser interferometer was used to detect both longitudinal and Rayleigh waves in fatigued Al2024 and as a 2D imaging system to spatially measure $\beta$ using surface waves [81]. A similar measurement with a heterodyne laser interferometer was utilized to make SHG measurements of Rayleigh waves in a nickel superalloy [38]. Recent developments in laser instrumentation such as the CHeap Optical Transducer (CHOT) [111,112] where patterns are essentially deposited on the surface of a sample for laser generation or transmission, could eventually make laser interrogation portable and feasible in an in situ application. Laser-based generation and detection has also been utilized in vibro-acoustic measurements [112-114]. Signals from a laser detector are usually weak such that careful signal conditioning is essential to reliably extract the second harmonic signal. 
Fig. 7 Normalized second harmonic Rayleigh wave amplitude measured over increasing propagation distance, on samples with varying intensities of shot-peening (reproduced from [80] with permission from Elsevier)

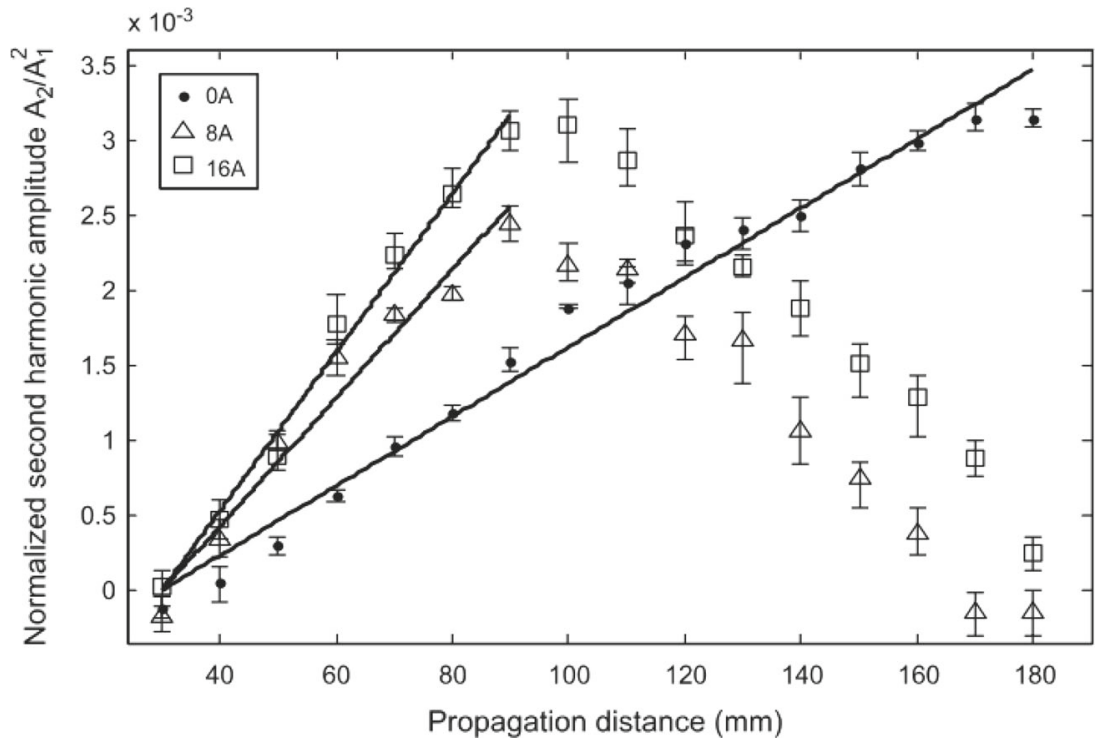

\subsection{Nonlinear Rayleigh Wave Measurements}

Surface wave measurements of the acoustic nonlinearity parameter have the advantage of reducing coupling and source effects of the transmitting source, as well as eliminating any potential nonlinearities induced by different excitation voltages. As such, measurements utilizing Rayleigh waves have received considerable attention throughout the literature-for example using a variety of wedge-contact generation and/or reception $[38,42,43,79$, 80,115-118], laser interferometer detection [38,81,115], air coupled detection [64], comb transducer generation and detection [39], and electromagnetic acoustic transducer (EMAT) detection $[119,120]$. While guided waves do offer some advantages in nonlinear measurements, achieving consistent coupling becomes increasingly difficult for guided wave wedge set-ups, which can still factor into measurement error on the detection side. So, the push toward a non-contact detection method will be crucial for robust measurements on real structures.

A nonlinear Rayleigh wave measurement typically measures the first and second harmonic wave amplitude at different propagation distances from the source transducer. A relative $\beta$ parameter, similar to that in Eq. (31), is generally made for Rayleigh waves, i.e.

$\beta_{R}^{\prime}=\left[\frac{A_{2}^{v}}{\left(A_{1}^{v}\right)^{2} x}\right] \frac{i 8 p}{\kappa_{P}^{2} \kappa_{R}}\left(1-\frac{2 \kappa_{R}^{2}}{\kappa_{R}^{2}+s^{2}}\right)$

Within a range of propagation distances, the amplitude ratio $A_{2}^{v} /\left(A_{1}^{v}\right)^{2}$ is approximately linearly proportional to $x$, and thus the slope is a proportional measure of $\beta^{\prime}$ from Eq. (35). Note that $\beta^{\prime}$ is also proportional to the material nonlinearity parameter, e.g. from Eqs. (4) or (19). An example of this linear fit between the normalized second harmonic amplitude and propagation distance is shown in Fig. 7, which shows measurements on samples with varying intensities of shot peening [80]. As seen in this example, the linear increase of $A_{2}^{v} /\left(A_{1}^{v}\right)^{2}$ over $x$ is only applicable over a range of $6 \mathrm{~cm}$ in two of the measurements, and about $11 \mathrm{~cm}$ in another sample, due to attenuation and diffraction effects. Care must be taken to account for these attenuation and diffraction effects, particularly if these effects change with damage imposed on the samples. Diffraction models for the nonlinearly generated second harmonic wave have been well developed for Rayleigh wave propagation from a variety of excitation sources [35,78], and recent work has further considered effects of nonlinearity from generating sources on nonlinear Rayleigh wave measurements $[40,64]$. This aspect is crucial for longer propagation distances, since the linear increase of the normalized second harmonic generated wave is only applicable for short propagation distances. Some work has relied on handselecting the linear region of $A_{2}^{v} /\left(A_{1}^{v}\right)^{2}$ over $x$, and extracting the slope of that linear fit to represent $\beta^{\prime}$. However, fitting models based on a least-squares fit have been applied to nonlinear Rayleigh wave measurements to more accurately extract $\beta^{\prime}$ by directly incorporating attenuation and diffraction [39]. More recently, these models have been expanded to incorporate nonlinear least squares curve-fitting algorithms to more accurately extract $\beta^{\prime}$ based not only on attenuation and diffraction effects, but also accounting for source nonlinearity effects [40]. A comparison of the raw linear fit of $A_{2}^{v} /\left(A_{1}^{v}\right)^{2}$ over $x$ and the nonlinear fitting algorithm to experimental nonlinear Rayleigh wave measurements is shown in Fig. 8, showing a wide range of propagation distances for which the nonlinear curve fitting can be applied. 


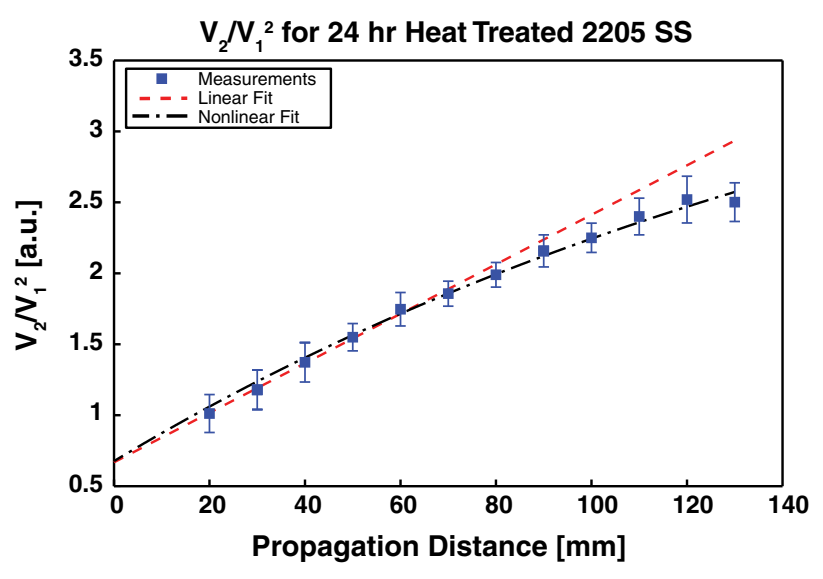

Fig. 8 Comparison of nonlinear diffraction curve-fit model and linear fit of normalized second harmonic amplitude over propagation distance from Rayleigh wave measurements on thermally aged 2205 duplex stainless steel [43] (reproduced from [40] with permission from Elsevier)

Many aspects of the experimental set-up for nonlinear Rayleigh wave measurements are similar to that of the contact longitudinal methods. A sinusoidal wave is excited in the source transducer with a high-power gated amplifier. A longer excitation signal is generally preferred, since this results in a longer steady state received signal. This facilitates accurate signal processing such that the instrumentation response (e.g. transient response of the transducers) can be removed before processing (FFT). Thus, for Rayleigh waves, around $20-30$ cycles is generally employed. The time domain signal is detected at some distance from the generating source and recorded with a digital oscilloscope or other data acquisition system, and averaged over many signals, generally around 256-512 signals to improve the signal-tonoise ratio. The signal is windowed and then the frequency response extracted with an FFT. From this, the first and second harmonic amplitudes can be determined. This process is then repeated at increasing propagation distances, keeping the input parameters constant and generally leaving the source transducer (and its coupling conditions) intact. Then, the source transducer is removed completely and remounted to repeat the entire measurement set multiple times to achieve statistically significant data. As shown in the example measurements in Figs. 7 and 8, multiple measurements at each propagation distance are averaged and one standard deviation from the mean are shown in error bar form attached to each data point. Since variations in nonlinear measurements can be quite large, failing to report this error analysis greatly discredits the work.

A variety of source and receiver configurations have been successfully employed for nonlinear Rayleigh wave measurements-specifically, wedge-contact generation and/or reception $[38,42,79,80]$, laser interferometer detec-

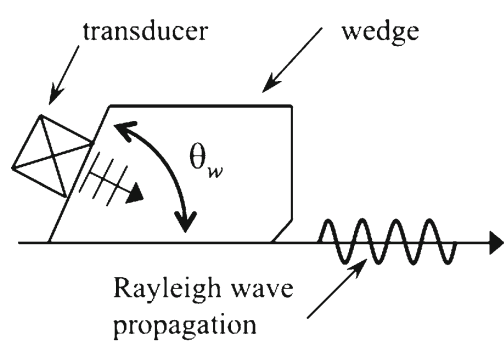

Fig. 9 Rayleigh wedge geometry

tion, comb transducer generation and detection [39], electromagnetic acoustic transducer (EMAT) detection [119,120], and air coupled detection [41,64]. The following sections discuss in detail some of these configurations.

\subsubsection{Wedge-Contact Methods}

Wedge-contact methods utilize an intermediary layer between the transducer and the sample to satisfy phase matching conditions. The wedge material is typically made of acrylic or other plastic material, and is designed to mount the transducer at the angle required to excite the Rayleigh surface wave in the sample. The wedge angle, $\theta_{w}$, as depicted in Fig. 9, can be determined from Snell's law:

$\sin \theta_{w}=\frac{c_{w}}{c_{R}}$

where $c_{w}$ is the longitudinal wave velocity of the wedge, and $c_{R}$ is the Rayleigh wave velocity in the sample. Acoustic coupling is necessary between both the transducer and wedge, as well as between the wedge and sample. Consistency in the clamping force on the transducer to the wedge, as well as on the wedge to the sample is also important. However, consistent clamping force and uniform contact are difficult to secure and this is the major source of scatter in this method.

\subsubsection{Air Coupled Detection}

Using air-coupled transducers for ultrasonic measurements is not new, e.g. [121-125], but only recently have they been applied to second harmonic generation measurements [41,64]. These methods have shown considerable improvement over wedge-contact reception methods since variations in contact conditions on the receiving end are essentially eliminated with the air-coupled detector. The air-coupled transducer detects a longitudinal wave in air that is leaked from the propagating Rayleigh wave in the sample. This component can be determined through displacement continuity considerations by relating the out-of-plane displacement of the Rayleigh wave to the longitudinal wave displacement in air $[41,126]$. One possible measurement setup is shown in Fig. 10 [41]. The main components of the air-coupled detec- 


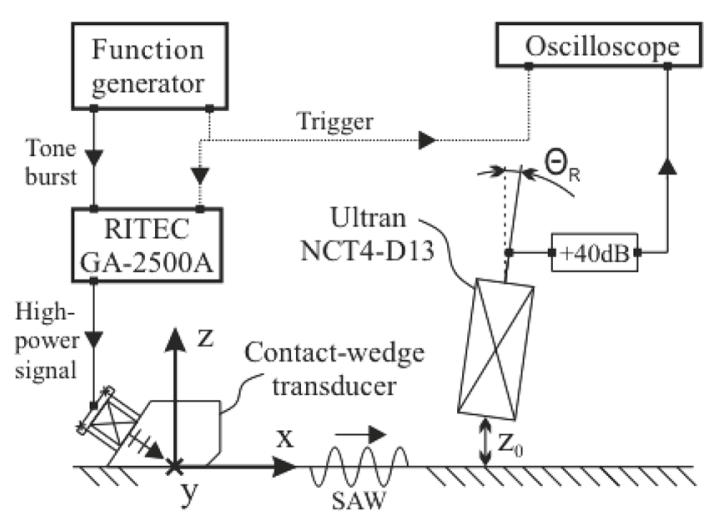

Fig. 10 Experimental setup of nonlinear Rayleigh wave measurement with air-coupled transducer detection (reproduced from [41] with permission from Elsevier)

tion system are the air-coupled transducer, which is mounted a vertical distance $z$ from the sample surface and at an angle $\theta_{R}$ to accurately detect the leaked Rayleigh wave, and a pulser-receiver to amplify the received signal. As in other measurement systems, the received signal is averaged over multiple signals to further improve the SNR.

The liftoff distance, which is defined as the vertical distance between the midpoint of the active area of air-coupled transducer and the sample ( $z$ as shown in Fig. 10), should be as small as possible to reduce attenuation and diffraction effects in air. This distance should be kept as consistent as possible throughout the measurements, or else the variation must be accounted for-the attenuation coefficient of ultrasonic waves in air is much higher than in metals, so small variations in $z$ could greatly influence the amplitude of the received wave. As such, a stable fixture for the air-coupled transducer is required. Similarly, local areas of surface inhomogeneity, curvature, or surface roughness could change the local liftoff distance, so care should be taken to ensure the sample surface is polished flat; otherwise, these variations must be characterized and accounted for.

Further details on this experimental setup can be found in [41,64]. Note that air-coupled transducers have not yet been used on the transmitting end of a second harmonic generation experiment. While the output of these transducers can generate a high enough stress wave for second harmonic generation in metals, the nonlinearity in air is so much larger than in metals that the nonlinear response of the air would dominate, masking the nonlinear response from the metal.

\subsection{SHG Measurement Variations and Corrections}

Variations in SHG measurements can arise from multiple sources [102]. These can include differences in coupling conditions between transducers and/or fixtures and the sample, variations in clamping force for affixing transducers the sam- ple. To accurately detect small changes in amplitude inherent in SHG measurements, digitizers in the detection system (oscilloscope or other $\mathrm{A} / \mathrm{D}$ converter) must have high enough resolution. Reflections from sample boundaries will influence the measured $\beta$ [127], so reflections in the received signal from long signals should be avoided or accounted for in analyses. Multiple measurements should be made by completely removing and remounting all fixtures from the sample and variations in measurements should be reported in all SHG results. A comprehensive study on effects of system nonlinear and measurement variations from positioning of transducers, coupling effects, and signal processing can be found in [102], along with experimental recommendations to minimize measurement variations.

Care must also be taken in terms of post-processing of measurements. For Rayleigh waves as described above, it is crucial to account for attenuation and diffraction of the first and second harmonic waves. For longitudinal waves, losses such as attenuation must be accounted for if they are non-negligible in the material and for the frequencies and propagation distances measured. In some measurements, frequency filtering of the first and second harmonic waves is crucial. In absolute measurements this is critical in extracting accurate values of $\beta$. In relative measurements, this is needed for example if different transducers are used in different measurements- the frequency response of transducers will bias the first and second harmonic differently.

\section{Applications}

There has been significant work in the past few decades aimed at using second harmonic generation as an NDE technique for early damage detection, by relating the acoustic nonlinearity parameter to different microstructural features. The basic models that relate the change in $\beta$ to the evolution of different microstructural features were reviewed in Sect. 2. This section reviews the literature that applies SHG measurements to monitor fatigue- and dislocation-based damage, thermal aging, and radiation damage.

\subsection{Fatigue- and Dislocation-Based Monitoring}

Due to the strong link between second harmonic generation and dislocations, a significant amount of work has focused on monitoring fatigue damage with second harmonic generation measurements. This problem was first considered from the standpoint of fatigue crack contribution to harmonic generation e.g. $[118,128]$, and later expanded to consider dislocation motion, the development of dislocation substructures [129], and dislocation dipole contribution to $\beta$ [45]. Cantrell later developed a more comprehensive theoretical model to relate the change in magnitude of $\beta$ due to a combi- 
nation of lattice anharmonicity, dislocation monopoles, dislocation dipoles, and their substructures (e.g. persistent slip bands, veins, channels) [51] that evolve during fatigue damage. These detailed models relate $\beta$ to percent of fatigue life in wavy slip metals (e.g. in polycrystalline nickel [46] and Al 2024 [51]), and 410Cb martensitic stainless steel [130]. These models have also been modified and applied to planar slip metals (e.g. IN100) [63], since the basic dislocation structures that form during fatigue in both types of materials are based on dislocation monopoles and dipoles. A structural health monitoring method for fatigue damage assessment based in part on second harmonic generation measurements and a probabilistic damage procedure [131] has been developed to predict fatigue crack initiation. In a later paper, Kulkarni and Achenbach [132] combined this crack initiation prediction procedure with a fatigue crack growth prediction model that incorporates imperfect inspections. A shift in focus to this type of monitoring with SHG could help advance the possibility of in-service applications of SHG measurements.

Extensive experimental work has used SHG measurements of $\beta$ to monitor fatigue damage with a variety of wave types and in a variety of materials, such as aluminum alloys $[45,51,117,118,129]$, nickel-based superalloys $[38,63,74]$, carbon steel $[42,119,133]$, stainless steel [134], titanium alloys $[79,81,86]$, Inconel [79], and single crystal copper [62]. Numerous experiments have shown that $\beta$ increases with increasing number of fatigue cycles and increasing cumulative plastic strain e.g. $[62,74,86,134]$. The acoustic nonlinearity parameter has shown to be more sensitive to fatigue damage than ultrasonic linear parameters such as wave velocity and attenuation throughout the earlier stages of fatigue [86], which has also been shown by Nagy in 2090 aluminum with acousto-elasticity nonlinear measurements [17] (note that this paper also measured nonlinearity in nonmetallic materials).

Monotonic increases in $\beta$ over fatigue life have been the most prevalent trend throughout the literature. Note that a clear overarching trend of $\beta$ over fatigue life is not realistic, due to complex dislocation structures that form differently over different types of fatigue conditions and material microstructures. Cantrell and Yost measured a monotonic increase in $\beta$ throughout fatigue in Al-2024 T4, which was in good agreement with their theoretical model that considered dislocation monopole and dipole contributions to $\beta$ [45]. Frouin et al. [86] measured acoustic nonlinearity in fatigued Ti-6Al-4V, showing $\beta$ increased by 180 up to $40 \%$ of fatigue life. Barnard et al. [79] measured $\beta$ in a Ti and Ni alloy during low and high cycle fatigue-measurements on the $\mathrm{Ni}$ alloy showed a general trend of increasing $\beta$ over increasing fatigue life, though noise in the data obscured the results. Kim et al. [74] measured an increase in $\beta$ in nickel superalloys throughout low cycle fatigue, and a slower and noisier increase in $\beta$ throughout high-cycle fatigue life with longitudinal waves. The trend of measured $\beta$ in the same nickel superalloy was found to be similar in Rayleigh wave measurements [38], indicating the change in acoustic nonlinearity parameter is independent of wave type used. Note that a similar trend of an initial sharp increase in $\beta$ followed by saturation at higher fatigue cycles was also seen with nonlinear Lamb waves in an Al-1100 alloy [84]. Measurements in A36type steel under low cycle fatigue also showed a monotonic increase in $\beta$, which also followed a similar trend to measured strain over increasing number of fatigue cycles [42]. Most recently, Apple et al. [62] measured $\beta$ in fatigued single crystal copper, and measured dislocation density and loop length using TEM analysis. Experimental results roughly showed a dependence of $\beta$ on the square root of cumulative plastic strain.

Two peaks in the measured nonlinearity over fatigue life have been measured in a few cases. Ogi et al. [119] reported on EMAT measurements of $\beta$ on fatigued steel rods, and saw an increase in second harmonic amplitude with increasing fatigue cycles up to about $60 \%$ fatigue life, where the authors reported $A_{2} / A_{1}$ as the nonlinearity, as opposed to the conventional definition of nonlinearity, $\left(A_{2} / A_{1}^{2}\right)$. Rao et al. [117] measured $\beta$ using Rayleigh waves throughout lowcycle fatigue of Al-7075, also showing two peaks in nonlinearity throughout fatigue life. Their thorough study considered three different samples under the same fatigue conditions, for two different sets of fatigue parameters. This dualpeak in $\beta$ over fatigue life was similar to that observed by Ogi et al. [119]. Although, the authors did not account for attenuation effects in their measurements of second harmonic generation, and the measured attenuation coefficient was shown to peak around the same percentage of fatigue life as the second SHG peak. A different trend was observed by Kumar et al. [135], where an ultrasonic fatigue system was adapted to make nonlinear measurements. This study and others showed a correlation between measurements and fatigue behavior in different aluminum alloys $[136,137]$. However, the relationship between $\beta$ and the measured second harmonic wave generated from this resonance system was not used. This relationship has actually been developed previously, for both classical nonlinearity from a standing wave system as well as hysteretic nonlinearity [138].

Detection of other dislocation-based damage mechanisms, aside from fatigue, has been demonstrated with SHG. The sensitivity of $\beta$ to cold work, which produces a significant amount of dislocations, along with residual stresses has been shown by Viswanath et al. [58] in measurements in cold-worked 304 stainless steel. The results showed a positive correlation of $\beta$ with percent cold work, and also with yield strength and tensile strength. Results were explained in terms of dislocations and dislocation substructures formed during cold rolling. Cold work and residual stresses due 
to surface shot peening has also been shown to increase $\beta$ measured with Rayleigh waves in Al-7075 [80]. Measurements of $\beta$ have also been correlated with increasing carbon content in quenched steel specimens [99], which was attributed to increasing amounts of dislocations with carbon content and interpreted using the Hikata et al. model [4]. Shui et al. [139] used SHG measurements to track monotonic loading effects on an AZ31 magnesium-aluminum alloy using longitudinal waves in different locations. The authors interpreted the experimental results with a previously developed microplasticity model [140]. Herrmann et al. [38] also looked at effects of monotonic loading in a nickel superalloy on the acoustic nonlinearity parameter using Rayleigh waves. Results showed an increase in $\beta$, where the increase was much greater in the initial stages of applied stress in monotonic loading. Rao et al. [141] measured $\beta$ in Al-7175 samples that were deformed under strain-controlled tensile tests, and compared results to X-ray diffraction measurements. Results showed that $\beta$ was location-dependent along the gauge length.

While the strong link between dislocation-based microstructural evolution and the acoustic nonlinearity parameter has been well-established throughout numerous experiments and developed models, care must be taken when applying similar analyses to interpret $\beta$ measurements on different materials under different damage conditions. For example, dislocation monopoles were shown to be the dominant mechanism of nonlinearity in fatigued single copper crystals [62], but as pointed out by Apple et al., this does not apply for other wavy slip metals such as Al-2024 or planar slip metals. Analysis is further complicated by the fact that techniques e.g. TEM capable of determining microstructural parameters such as dislocation density and dislocation loop lengths can have substantial variation. This variation in measured parameters has been reported to propagate out to a standard error in theoretical calculations of $\beta$ of up to $70 \%$ [62]. Further, TEM measurements only look at a volume of material that is orders of magnitude smaller than typically insonified volumes for SHG measurements. The key aspect to interpreting these dislocation-based SHG measurements, as shown particularly in the numerous SHG measurements over fatigue damage, is that specific dislocation substructures and evolution in the material system measured is crucial for accurate SHG measurement evaluation.

\subsection{Thermal Aging Monitoring}

Significant work has considered the effect of microstructural features that evolve throughout thermal aging, such as precipitates, vacancies, and voids, to the acoustic nonlinearity parameter. This has potential applications in monitoring overaging or embrittlement of critical structural components at elevated temperatures. Previously explored materials range from aluminum alloys [47,48, 129, 142], Inconel alloys [33, 100], titanium alloys [97,143], ferritic steels [49, 101,144], stainless steels [43], and maraging steels [87,145].

Second harmonic generation due to precipitation during thermal aging was first suggested by Yost and Cantrell [129]. Initial experimental evidence showed an increase of about $10 \%$ in $\beta$ throughout the heat treatment of Al-2024 from the T4 to T6 temper. They pointed out that the change in $\beta$ due to fatigue was much greater than changes due to precipitates, but that precipitate structures could greatly affect the dislocation structure and interactions during fatigue. Further exploration by Barnard et al. [33] on thermally embrittled Inconel 718 showed that with increasing heat treatment time, $\beta$ increased then decreased in one heat, and only decreased in the other heat investigated. However, the increase in $\beta$ was perhaps only a result of a decrease in $A_{1}$, since at this measurement $A_{2}$ was below the noise floor of the signal. Results were compared to small punch test results, and changes in $\beta$ during heat treatments were attributed to coherent second phase precipitates that formed and ultimately lost coherency with the matrix, and differences in $\beta$ between the two materials were attributed to platelet phases that formed in one material and not the other-potential evidence that grain structure influences nonlinearity.

Theoretical models were developed that related changes in $\beta$ to coherency strains in the matrix [142], which were due to precipitates in the matrix. Experiments showed that $\beta$ was proportional to the volume fraction of precipitates and the effective misfit of the precipitate in the matrix. Cantrell and Zhang [47] further modified this model to incorporate dislocation and precipitate interactions to describe changes in $\beta$. Yet another model was developed to relate both the growth of precipitates and nucleation of precipitates to $\beta$ [48]. This model was then compared to experimental measurements of $\beta$ (from [129]) measured over precipitation heat treatment time in Al-2024 from the T4 to T6 temper. Results were consistent with the fact that most precipitates nucleate within the first portion of heat treatment time in $\mathrm{Al} 2024$, and showed an increase in $\beta$ after short aging times, then a decrease in $\beta$ (to below the value in the unaged state) with increasing aging time, and then a second increase in $\beta$ at even longer aging times. While supported by experimental evidence, these precipitate-pinning models assume the stress field of all the precipitates influence some dislocation segment at the low stress amplitudes of SHG measurements, which for materials with low dislocation density or low precipitate volume fractions might not be the case.

Measurements of $\beta$ have been made in a variety of materials subjected to thermal aging. Hurley et al. [49] measured $\beta$ in ASTM A710 steel that was heat treated to produce varying amounts of precipitate hardening. Results were compared to inhomogeneous strain. The paper shows a positive correlation between $\beta$ and strain, but only results from one 
sample set was convincing - the results from the second sample set does not show a clear linear trend between $\beta$ and strain. A modified model from [47] is used to express the contribution of $\beta$ from precipitate and dislocation interactions, but experimental results could not be compared to this model since the authors were unable to measure dislocation density.

Measurements of $\beta$ in heat treated $\mathrm{CrMoV}$ have been correlated to fracture toughness measured from Charpy impact experiments [146]. The authors attribute the change in $\beta$ to segregation of impurity elements to grain boundaries, but they provide no theoretical or physical basis for the contribution of this effect to nonlinearity. Park et al. [147] measured $\beta$ over isothermal aging and creep of $9-12 \mathrm{Cr}$ ferriticmartensitic steel and compared measurements to metallurgical analyses. Results consistently showed a drastic decrease in $\beta$ followed by a gradual increase, over different aging temperatures, which the authors correlated to changes in dislocation density and second phase distributions. Abraham et al. [148] correlated an increase in $\beta$ with sensitized 304 stainless steel subjected to intergranular corrosion. Note that Lamb waves have also been utilized to measure $\beta$ during thermal aging of a ferritic Cr-Ni steel [149].

Metya et al. [145] studied SHG in thermally aged C-250 maraging steel, showing a maximum increase of $\beta$ at peak hardening. Results were interpreted with X-ray diffraction and TEM results, showing $\mathrm{Ni}_{3} \mathrm{Ti}$ and $\mathrm{FeTi}$ precipitate formation followed by coarsening with increasing heat treatment times. Unfortunately, the authors do not mention the measurement error for $\beta$, which greatly weakens the paper's conclusions. In a separate work, Viswanath et al. [87] measured $\beta$ in M250 grade maraging steel subjected to thermal aging of varying times. Results show a linear increase in $\beta$ during the middle stages of heat treatment time, which the authors explain is due to precipitation of $\mathrm{Ni}_{3} \mathrm{Ti}$ that causes microstrain. The authors used a prior theoretical model [142] to explain their results. Work by $\mathrm{Li}$ et al. [100] measured $\beta$, attenuation, and ultrasonic velocity in heat treated Inconel X750. Results showed that $\beta$ was more sensitive to microstructural changes than linear measurements - the change in $\beta$ was up to $70 \%$ compared to a $16 \%$ change in attenuation and $0.8 \%$ change in ultrasonic velocity.

Recent work by Ruiz et al. [43] measured $\beta$ over increasing thermal damage in 2205 duplex stainless steel, as well as attenuation, and ultrasonic velocity. Results showed the nonlinearity parameter was more sensitive to thermal aging than the linear parameters $-\beta$ increased during the initial aging time, then decreased and increased with increasing aging time. Changes in $\beta$ were attributed to the increasing formation of the sigma phase over increasing time of thermal aging as measured by SEM analysis, results of which correlate to degradation of impact properties of the stainless steel. Nucera et al. [98] developed a model for the effect of thermal stresses on second harmonic generation with varying temperature loads. Their model incorporates the anharmonic interatomic potential of energy absorbed during an applied temperature load. Experimental results confirm an increase in $\beta$ as a result of an increase in temperature of a constrained steel sample, although the authors did not consider all other effects from experimental factors and contributors to nonlinearity in the analysis.

In summary, microstructural evolution throughout thermal aging of metals has shown to be detectable with SHG measurements. However, differences among different samples such as texture or preferred grain orientations in a material microstructure have also been shown to influence $\beta$. In a study on a heat treated titanium alloy, Mukhopadhyay et al. [143] correlated cooling rates with scatter in the nonlinearity parameter, attributing measurement variation to texture, grain size, and grain orientation. So, care must be taken to characterize and account for these variations to unambiguously link $\beta$ with microstructural changes that are early indications of thermally-induced material degradation. Further, it is crucial to relate measurements of $\beta$ to microstructural features (e.g. precipitate formation) and then to macroscopic damage (e.g. embrittlement). Different microstructural features can lead to the same type of material degradation, and since $\beta$ is sensitive to microstructural features, this link cannot be skipped.

\subsection{Creep Damage}

Creep damage has also been studied using SHG measurement techniques, which are realized due to the combination of dislocations, precipitates, and voids that form during creep. Baby et al. [97] measured the acoustic nonlinearity parameter in a titanium alloy subjected to creep damage, which produced an increase of volume fraction of voids in the microstructure. Results showed an increase in $\beta$ up to about $60 \%$ of creep life, followed by a decrease in $\beta$, with a maximum of about a $200 \%$ increase. They attributed the increase in $\beta$ to the increase in volume fraction of microvoids, and the decrease in $\beta$ to an increase in the damage scale caused by coalescence of the microvoids as seen by optical microscopy.

Balasubramanian et al. [150] studied changes in $\beta$ (along with the third harmonic and static components) in creep fracture pure copper specimens using a low amplitude excitation method based on [106]. However, it is difficult to discern meaning from the results due to the lack of thorough discussion on the complex creep testing matrix compared with the ultrasonic measurements, and their newly developed measurement technique of utilizing low amplitude excitation showed an error of up to $50 \%$. Still, results indicate that a more accurate measurement of $\beta$ at low drive amplitudes could provide useful microstructural information relating to creep damage. Valluri et al. [151] and Narayana et al. [152] reported measurements of the static component and second 
and third harmonic amplitudes at the typical higher excitation amplitudes in pure copper material in continuous and interrupted creep-induced damage. Measurements showed a greater sensitivity of the third harmonic to creep damage.

Kim et al. [153] measured $\beta$ in IN738 alloys subjected to high-temperature creep. Measurements were made at multiple time intervals leading up to failure, in comparison to field emission scanning electron microscopy of $\mathrm{Ni}_{3} \mathrm{Al}$ precipitates. Results showed a monotonic increase in $\beta$ with increasing fraction of fatigue life for specimens under different static loads; unfortunately, $\beta$ measurement error is not reported, again, weakening the paper's conclusions. Note that the previously discussed paper of Park et al. [147] monitored creep damage as well, indicating the applicability of SHG to detect early stage creep damage.

\subsection{Radiation Damage}

Recently, second harmonic generation measurements have been used to monitor radiation damage in structural material [75,76,154]. Actually, an early work measured second harmonic generation in neutron irradiated copper single crystals [2]. These copper single crystals were annealed and neutronirradiated to $3.6 \times 10^{15} \mathrm{n} / \mathrm{cm}^{2}$. Results showed a decrease in second harmonic amplitude as well as in measured attenuation due to neutron bombardment, which the authors postulated was caused by the pinning or immobilization of dislocations in the irradiated material. Current work $[75,76]$ has focused on detecting radiation-induced embrittlement, a result of microstructural features such as precipitate phases, dislocations, and defect clusters, in nuclear reactor pressure vessel steel material [155]. Note in comparison to the irradiated copper single crystals, the neutron fluence for reactor pressure vessel materials is about $1-3 \times 10^{19} \mathrm{n} / \mathrm{cm}^{2}(\mathrm{E}>$ $1 \mathrm{MeV}$ ) after 40 years of operation, and significantly higher for internals materials closer to the fuel rods. Note that the explanation offered by Cash and Cai [55] that the decrease in $\beta$ due to irradiation in single crystal copper in [2] is possibly due to the reduction of dislocation dipole contribution to $\beta$ does not apply to irradiated RPV steels, since dislocation dipoles do not form during irradiation and are extremely unlikely to be present in the as-annealed materials.

Recent work shows that the acoustic nonlinearity parameter is sensitive to microstructural changes in reactor pressure vessel steels induced by increasing neutron fluence, different irradiation temperatures, and different material compositions [75,76]. Similar trends of $\beta$ over neutron fluence were measured in two separate low-alloy steel materials, and $\beta$ increased up to a roughly $5 \times 10^{19} \mathrm{n} / \mathrm{cm}^{2}$ followed by a decrease. A model for the change in $\beta$ due to precipitate formation over different irradiation parameters generally agreed with experimental results.

\subsection{Other Damage Types}

SHG techniques have been utilized for a variety of other types of damage. Rayleigh waves have been used to interrogate coating damage [116], although no evidence of coating damage over the stress levels was provided. In another work, Rayleigh waves were used to monitor stress corrosion cracking in carbon steel, over increasing applied stress [156]. Results were consistent with the theory of nonlinearity generated by cracks developed by Nazarov and Sutin [52].

There has been extensive work done on monitoring cracking in a variety of materials with SHG techniques. The phenomenon of contact acoustic nonlinearity, directly related to nonlinearity generated from crack interfaces, has been utilized to interrogate material interfaces $[50,128,157]$ and bonds $[158,159]$. This contact acoustic nonlinearity has shown to be much stronger than material nonlinearity [160]. SHG has been used to monitor for example hydration processes in concrete [161], but SHG in concrete has not been widely realized as of yet due to high attenuation effects of the second harmonic in this material.

\section{Conclusions and Future Outlook}

Second harmonic generation measurement techniques have the unique capability to detect microstructural changes in metals prior to macroscopic cracking. While these methods have been studied for many decades, they have received considerable attention in the recent literature in efforts to address safe and effective operation of the aging infrastructure of transportation, energy industries, and defense systems. This article reviews the different microstructural contributions to the acoustic nonlinearity parameter $\beta$, and the variety of measurement systems developed to date for longitudinal wave and Rayleigh wave SHG measurements of $\beta$. Applications of $\beta$ to fatigue and other dislocation-based material damage, thermal aging, creep damage, and radiation damage are discussed.

Limitations should be kept in mind with any SHG measurement technique. Scatter in SHG measurements is a welldocumented issue, and can be considerable if care is not taken during experimentation to eliminate, minimize, or account for all sources of variation (set up, contact conditions, sample preparation, texture, etc.). The inherent scatter in measurements of dislocation density and loop lengths in microscopy methods such as TEM complicate the comparison of experiment to theory. Some microscopy techniques also only look at an extremely small region of the material compared to the volume of the interrogating ultrasonic wave. So, any local heterogeneity should be considered during analysis of SHG measurements, since SHG is a global average of the interrogated volume of material. 
In terms of future thrusts with SHG measurement techniques, the development of air-coupled and laser-based detection systems will be crucial for monitoring real components and structures. In real components, surface conditions can be extremely variable and direct surface access is not necessarily possible. At the same time, it is necessary to continue to push the controlled laboratory-type measurements to better understand SHG in terms of microstructural changes and effects, and to continue to develop physics-based materials models to relate nonlinear ultrasonic wave propagation to material microstructural changes. The thrust of the extensive model development relating SHG to microstructural evolution throughout fatigue $[46,51,63,140]$ serve as a guide for future model development for other microstructural contributions to $\beta$, and should be reciprocated in other damage types. Further, absolute measurements of SHG have only been realized in controlled laboratory settings, and are not yet feasible for anywhere near an in-situ measurements. And, components that SHG measurements could be applied to and useful for will most likely not have a baseline (i.e. $\beta_{0}$ ) measurement of SHG to compare, which is absolutely necessary for the interpretation of relative measurements of $\beta$. So, either the development of a system for a practical absolute measurement of $\beta$ is needed, or a calibration system will need to be developed. SHG measurement systems that can be incorporated on components from the start of operation may be a promising focus for newly designed structures. Finally, there is substantial evidence that $\beta$ can monitor microstructural evolution leading to macroscopic damage. However, studies using $\beta$ to predict remaining useful life or damage of structural components has been limited. A focus on this aspect would have extremely important applications in the area of structural health monitoring $[131,132]$.

Acknowledgments The authors would like to acknowledge funding received from the DOE Office of Nuclear Energy's Nuclear Energy University Programs.

\section{References}

1. Breazeale, M.A., Thompson, D.O.: Finite-amplitude ultrasonic waves in aluminum. Appl. Phys. Lett. 3, 77-78 (1963)

2. Breazeale, M.A., Ford, J.: Ultrasonic studies of the nonlinear behavior of solids. J. Appl. Phys. 36, 3486-3490 (1965)

3. Gedroitz, A.A., Krasilnikov, V.A.: Elastic waves of finite amplitude and deviationsfrom Hook's law. Sov. Phys. JETP (Engl. Transl.) 16, 1122-1131 (1963)

4. Hikata, A., Chick, B.B., Elbaum, C.: Dislocation contribution to the second harmonic generation of ultrasonic waves. J. Appl. Phys. 36, 229-236 (1965)

5. Hikata, A., Elbaum, C.: Generation of ultrasonic second and third harmonics due to dislocations. I. Phys. Rev. 144, 469-477 (1966)
6. Gauster, W.B., Breazeale, M.A.: Ultrasonic measurement of the nonlinearity parameters of copper single crystals. Phys. Rev. 168, 655-661 (1968)

7. Yost, W.T., Cantrell, J.H., Breazeale, M.A.: Ultrasonic nonlinearity parameters and third-order elastic constants of copper between 300 and $3{ }^{\circ} \mathrm{K}$. J. Appl. Phys. 52, 126-128 (1981)

8. Thompson, D.O., Buck, O., Barnes, R.S., Huntington, H.B.: Diffusional properties of the stage-III defect in copper. I. Experimental results. J. Appl. Phys. 38, 3051-3056 (1967)

9. Thompson, D.O., Buck, O., Huntington, H.B., Barnes, P.S.: Diffusional properties of the stage-III defect in copper. II. A model for defect-dislocation interactions. J. Appl. Phys. 38, 3057 (1967)

10. Thompson, D.O., Buck, O.: Diffusional properties of the stageIII defect in copper. III. Bulk diffusion. J. Appl. Phys. 38, 3068 (1967)

11. Gauster, W.B., Breazeale, M.A.: Detector for measurement of ultrasonic strain amplitudes in solids. Rev. Sci. Instrum. 37, 15441548 (1966)

12. Thompson, R.B., Buck, O., Thompson, D.O.: Higher harmonics of finite amplitude ultrasonic waves in solids. J. Acoust. Soc. Am. 59, 1087-1094 (1976)

13. Yost, W.T., Breazeale, M.A.: Adiabatic third-order elastic constants of fused silica. J. Appl. Phys. 44, 1909-1910 (1973)

14. Hurley, D.C., Fortunko, C.M.: Determination of the nonlinear ultrasonic parameter $\beta$ using a Michelson interferometer. Meas. Sci. Technol. 8, 634-642 (1997)

15. Cantrell, J.H., Breazeale, M.A.: Ultrasonic investigation of the nonlinearity of fused silica for different hydroxyl-ion contents and homogeneities between 300 and 3K. Phys. Rev. B. 17, 48644870 (1978)

16. Cantrell, J.H.: Fundamentals and applications of nonlinear ultrasonic nondestructive evaluation. In: Kundu, T. (ed.) Ultrasonic Nondestructive Evaluation, pp. 363-434. CRC Press, Boca Raton (2004)

17. Nagy, P.B.: Fatigue damage assessment by nonlinear ultrasonic materials characterization. Ultrasonics 36, 375-381 (1998)

18. Guyer, R.A., Johnson, P.A.: Nonlinear mesoscopic elasticity: evidence for a new class of materials. Phys. Today 52, 30-36 (1999)

19. Payan, C., Garnier, V., Moysan, J., Johnson, P.A.: Applying nonlinear resonant ultrasound spectroscopy to improving thermal damage assessment in concrete. J. Acoust. Soc. Am. 121, EL125 (2007)

20. Van Den Abeele, K.E.-A., Carmeliet, J., Ten Cate, J.A., Johnson, P.A.: Nonlinear elastic wave spectroscopy (NEWS) techniques to discern material damage, part II: single-mode nonlinear resonance acoustic spectroscopy. Res. Nondestruct. Eval. 12, 31-42 (2000)

21. Ballad, E.M., Vezirov, S.Y., Pfleiderer, K., Solodov, I.Y., Busse, G.: Nonlinear modulation technique for NDE with air-coupled ultrasound. Ultrasonics 42, 1031-1036 (2004)

22. Van Den Abeele, K.E.-A., Johnson, P.A., Sutin, A.: Nonlinear elastic wave spectroscopy (NEWS) techniques to discern material damage, part i: nonlinear wave modulation spectroscopy (NWMS). Res. Nondestruct. Eval. 12, 17-30 (2000)

23. Anderson, B.E., Griffa, M., Larmat, C., Ulrich, T.J., Johnson, P.A.: Time reversal. Acoust. Today 4, 5-16 (2008)

24. Larmat, C.S., Guyer, R.A., Johnson, P.A.: Time-reversal methods in geophysics. Phys. Today 63(8), 31-35 (2010)

25. Croxford, A.J., Wilcox, P.D., Drinkwater, B.W., Nagy, P.B.: The use of non-collinear mixing for nonlinear ultrasonic detection of plasticity and fatigue. J. Acoust. Soc. Am. 126, EL117-EL122 (2009)

26. Liu, M., Tang, G., Jacobs, L.J., Qu, J.: Measuring acoustic nonlinearity parameter using collinear wave mixing. J. Appl. Phys. 112, 024908 (2012) 
27. Jhang, K.: Nonlinear ultrasonic techniques for non-destructive assessment of micro damage in material: a review. Int. J. Precis. Eng. Manuf. 10, 123-135 (2009)

28. Zheng, Y., Maev, R.G., Solodov, I.Y.: Review/synthèse nonlinear acoustic applications for material characterization: a review. Can. J. Phys. 77, 927-967 (2000)

29. Cantrell, J.H.: Crystalline structure and symmetry dependence of acoustic nonlinearity parameters. J. Appl. Phys. 76, 3372 (1994)

30. Norris, A.N.: Finite-amplitude waves in solids. In: Hamilton, M.F., Blackstock, D.T. (eds.) Nonlinear Acoust., pp. 263-277. Academic Press, San Diego (1998)

31. Shui, Y., Solodov, I.Y.: Nonlinear properties of Rayleigh and Stoneley waves in solids. J. Appl. Phys. 64, 6155 (1988)

32. De Lima, W.J.N., Hamilton, M.F.: Finite-amplitude waves in isotropic elastic plates. J. Sound Vib. 265, 819-839 (2003)

33. Barnard, D.J., Dace, G.E., Buck, O.: Acoustic harmonic generation due to thermal embrittlement of Inconel 718. J. Nondestruct. Eval. 16, 67-75 (1997)

34. Rogers, P.H., Van Buren, A.L.: An exact expression for the Lommel diffraction correction integral. J. Acoust. Soc. Am. 55, 724728 (1974)

35. Shull, D.J., Kim, E.E., Hamilton, M.F., Zabolotskaya, E.A.: Diffraction effects in nonlinear Rayleigh wave beams. J. Acoust. Soc. Am. 97, 2126-2137 (1995)

36. Ingenito, F., Williams, A.O.: Calculation of second-harmonic generation in a piston beam. J. Acoust. Soc. Am. 49, 319-328 (1971)

37. Zabolotskaya, E.A.: Nonlinear propagation of plane and circular Rayleigh waves in isotropic solids. J. Acoust. Soc. Am. 91, 25692575 (1992)

38. Herrmann, J., Kim, J.-Y., Jacobs, L.J., Qu, J., Littles, J.W., Savage, M.F.: Assessment of material damage in a nickel-base superalloy using nonlinear Rayleigh surface waves. J. Appl. Phys. 99, 124913 (2006)

39. Hurley, D.C.: Nonlinear propagation of narrow-band Rayleigh waves excited by a comb transducer. J. Acoust. Soc. Am. 106, 1782-1788 (1999)

40. Torello, D., Thiele, S., Matlack, K., Kim, J.-Y., Qu, J., Jacobs, L.J.: Diffraction, attenuation, and source corrections for nonlinear Rayleigh wave ultrasonic measurements. Ultrasonics 56, 417-426 (2015)

41. Thiele, S., Kim, J., Qu, J., Jacobs, L.J.: Air-coupled detection of nonlinear rayleigh surface waves to assess material nonlinearity. Ultrasonics 54, 1470-1475 (2014)

42. Walker, S.V., Kim, J.-Y., Qu, J., Jacobs, L.J.: Fatigue damage evaluation in A36 steel using nonlinear Rayleigh surface waves. NDT E Int. 48, 10-15 (2012)

43. Ruiz, A., Ortiz, N., Medina, A., Kim, J.-Y., Jacobs, L.J.: Application of ultrasonic methods for early detection of thermal damage in 2205 duplex stainless steel. NDT E Int. 54, 19-26 (2013)

44. Hikata, A., Sewell, F.A., Elbaum, C.: Generation of ultrasonic second and third harmonics due to dislocations II. Phys. Rev. 151, 442-449 (1966)

45. Cantrell, J.H., Yost, W.T.: Nonlinear ultrasonic characterization of fatigue microstructures. Int. J. Fatigue 23, S487-S490 (2001)

46. Cantrell, J.H.: Substructural organization, dislocation plasticity and harmonic generation in cyclically stressed wavy slip metals. Proc. R. Soc. Lond. Ser. A. 460, 757-780 (2004)

47. Cantrell, J.H., Zhang, X.-G.: Nonlinear acoustic response from precipitate-matrix misfit in a dislocation network. J. Appl. Phys. 84, 5469-5472 (1998)

48. Cantrell, J.H., Yost, W.T.: Determination of precipitate nucleation and growth rates from ultrasonic harmonic generation. Appl. Phys. Lett. 77, 1952-1954 (2000)

49. Hurley, D.C., Balzar, D., Purtscher, P.T.: Nonlinear ultrasonic assessment of precipitation hardening in ASTM A710 steel. J. Mater. Res. 15, 2036-2042 (2000)
50. Biwa, S., Hiraiwa, S., Matsumoto, E.: Experimental and theoretical study of harmonic generation at contacting interface. Ultrasonics 44, e1319-e1322 (2006)

51. Cantrell, J.H.: Quantitative assessment of fatigue damage accumulation in wavy slip metals from acoustic harmonic generation. Philos. Mag. 86, 1539-1554 (2006)

52. Nazarov, V.E., Sutin, A.M.: Nonlinear elastic constants of solids with cracks. J. Acoust. Soc. Am. 102, 3349-3354 (1997)

53. Suzuki, T., Hikata, A., Elbaum, C.: Anharmonicity due to glide motion of dislocations. J. Appl. Phys. 35, 2761 (1964)

54. Granato, A., Lücke, K.: Theory of mechanical damping due to dislocations. J. Appl. Phys. 27, 583-593 (1956)

55. Cash, W.D., Cai, W.: Dislocation contribution to acoustic nonlinearity: the effect of orientation-dependent line energy. J. Appl. Phys. 109, 014915 (2011)

56. Zhang, J., Xuan, F., Xiang, Y.: Dislocation characterization in cold rolled stainless steel using nonlinear ultrasonic techniques: a comprehensive model. Europhys. Lett. 103, 68003 (2013)

57. Zhang, J., Xuan, F.: A general model for dislocation contribution to acoustic nonlinearity. Europhys. Lett. 105, 54005 (2014)

58. Viswanath, A., Rao, B.P.C., Mahadevan, S., Parameswaran, P., Jayakumar, T., Raj, B.: Nondestructive assessment of tensile properties of cold worked AISI type 304 stainless steel using nonlinear ultrasonic technique. J. Mater. Process. Technol. 211, 538-544 (2011)

59. Chen, Z., Qu, J.: Dislocation-induced acoustic nonlinearity parameter in crystalline solids. J. Appl. Phys. 114, 164906 (2013)

60. Cash, W.D., Cai, W.: Contribution of dislocation dipole structures to the acoustic nonlinearity. J. Appl. Phys. 111, 074906 (2012)

61. Cantrell, J.H., Yost, W.T.: Acoustic harmonic-generation from fatigue-induced dislocation dipoles. Philos. Mag. A. 69, 315-326 (1994)

62. Apple, T.M., Cantrell, J.H., Amaro, C.M., Mayer, C.R., Yost, W.T., Agnew, S.R., Howe, J.M.: Acoustic harmonic generation from fatigue-generated dislocation substructures in copper single crystals. Philos. Mag. 93, 2802-2825 (2013)

63. Cantrell, J.H.: Ultrasonic harmonic generation from fatigueinduced dislocation substructures in planar slip metals and assessment of remaining fatigue life. J. Appl. Phys. 106, 1-6 (2009)

64. Thiele, S.: Air-coupled detection of Rayleigh surface waves to asses material nonlinearity due to precipitation in alloy steel. Master's Thesis, Georgia Institute of Technology (2013)

65. Eringen, A.C.: Mechanics of Continua. Robert E. Krieger Publishing Company, Huntington (1980)

66. Thiele, S., Matlack, K.H., Kim, J.-Y., Qu, J., Wall, J.J., Jacobs, L.J.: Assessment of precipitation in alloy steel using nonlinear Rayleigh surface waves. destructive Evaluation, vol. 1581, pp. 682-689. AIP Publishing (2014)

67. Martin, J.W.: Precipitation Hardening. Permagon Press, Oxford (1968)

68. Hirose, S., Achenbach, J.D.: Higher harmonics in the far field due to dynamic crack-face contacting. J. Acoust. Soc. Am. 93, 142-147 (1993)

69. Johnson, K.L.: Contact mechanics. Cambridge University Press, Cambridge (1985)

70. Greenwood, J.A., Williamson, J.: Contact of nominally flat surfaces. Proc. R. Soc. Lond. Ser. A. 295, 300-319 (1966)

71. Kim, J.-Y., Lee, J.-S.: A micromechanical model for nonlinear acoustic properties of interfaces between solids. J. Appl. Phys. 101, 043501 (2007)

72. Pecorari, C.: Adhesion and nonlinear scattering by rough surfaces in contact: beyond the phenomenology of the Preisach-Mayergoyz framework. J. Acoust. Soc. Am. 116, 1938-1947 (2004)

73. Breazeale, M.A., Philip, J.: Determination of third-order elastic constants from ultrasonic harmonic generation measurements. In: 
Mason, W.P., Thurston, R.N. (eds.) Physical Acoustics, vol. XVII, pp. 1-60. Academic Press, New York (1984)

74. Kim, J.-Y., Jacobs, L.J., Qu, J., Littles, J.W.: Experimental characterization of fatigue damage in a nickel-base superalloy using nonlinear ultrasonic waves. J. Acoust. Soc. Am. 120, 1266-1273 (2006)

75. Matlack, K.H., Wall, J.J., Kim, J.-Y., Qu, J., Jacobs, L.J., Viehrig, H.-W.: Evaluation of radiation damage using nonlinear ultrasound. J. Appl. Phys. 111, 054911 (2012)

76. Matlack, K.H., Kim, J.-Y., Wall, J.J., Qu, J., Jacobs, L.J., Sokolov, M.A.: Sensitivity of ultrasonic nonlinearity to irradiated, annealed, and re-irradiated microstructure changes in RPV steels. J. Nucl. Mater. 448, 26-32 (2014)

77. Adler, E.L., Bridoux, E., Coussot, G., Dieulesaint, E.: Harmonic generation of acoustic surface waves in $\mathrm{Bi} 12 \mathrm{GeO} 20$ and $\mathrm{LiNbO} 3$. IEEE Trans. Sonics Ultrason. 20, 13-16 (1973)

78. Shull, D.J., Hamilton, M.F., Il'insky, Y.A., Zabolotskaya, E.A.: Harmonic generation in plane and cylindrical nonlinear Rayleigh waves. J. Acoust. Soc. Am. 94, 418-427 (1993)

79. Barnard, D.J., Brasche, L.J.H., Raulerson, D., Degtyar, A.D.: Monitoring fatigue damage accumulation with rayleigh wave harmonic generation measurements. In: Thompson, D.O., Chimenti, D.E. (eds.) Review of Progress in Quantitative Nondestructive Evaluation, pp. 1393-1400. AIP, New York (2003)

80. Liu, M., Kim, J.-Y., Jacobs, L.J., Qu, J.: Experimental study of nonlinear Rayleigh wave propagation in shot-peened aluminum plates-feasibility of measuring residual stress. NDT E Int. 44, 67-74 (2011)

81. Blackshire, J.L., Sathish, S., Na, J.K., Frouin, J.: Nonlinear laser ultrasonic measurements of localized fatigue damage. In: Thompson, D.O., Chimenti, D.E. (eds.) Review of Progress in Quantitative Nondestructive Evaluation, pp. 1479-1488. AIP, New York (2003)

82. Bermes, C., Kim, J.-Y., Qu, J., Jacobs, L.J.: Experimental characterization of material nonlinearity using Lamb waves. Appl. Phys. Lett. 90, 021901 (2007)

83. Deng, M., Pei, J.: Assessment of accumulated fatigue damage in solid plates using nonlinear Lamb wave approach. Appl. Phys. Lett. 90, 121902-121903 (2007)

84. Pruell, C., Kim, J.-Y., Qu, J., Jacobs, L.J.: Evaluation of fatigue damage using nonlinear guided waves. Smart Mater. Struct. 18, 035003 (7 pp.) (2009)

85. Matlack, K.H., Kim, J.-Y., Jacobs, L.J., Qu, J.: Experimental characterization of efficient second harmonic generation of Lamb wave modes in a nonlinear elastic isotropic plate. J. Appl. Phys. 109, 014905 (2011)

86. Frouin, J., Sathish, S., Matikas, T.E., Na, J.K.: Ultrasonic linear and nonlinear behavior of fatigued Ti-6Al-4V. J. Mater. Res. 14, 1295-1298 (1998)

87. Viswanath, A., Rao, B.P.C., Mahadevan, S., Jayakumar, T., Baldev, R.: Microstructural characterization of M250 grade maraging steel using nonlinear ultrasonic technique. J. Mater. Sci. 45, 6719-6726 (2010)

88. Cantrell, J.H., Salama, K.: Acoustoelastic characterization of materials. Int Mater Rev. 36, 125-145 (1991)

89. Yost, W.T., Cantrell, J.H.: Anomalous nonlinearity parameters of solids at low acoustic drive amplitudes. Appl. Phys. Lett. 94, 021905 (2009)

90. Moreau, A.: Detection of acoustic second harmonics in solids using a heterodyne laser interferometer. J. Acoust. Soc. Am. 98, 2745-2752 (1995)

91. Yost, W.T., Cantrell, J.H., Kushnick, P.W.: Fundamental aspects of pulse phase-locked loop technology-based methods for measurement of ultrasonic velocity. J. Acoust. Soc. Am. 91, 1456-1468 (1992)
92. Dace, G.E., Thompson, R.B., Buck, O.: Measurement of the acoustic harmonic generation for materials characterization using contact transducers. In: Thompson, D.O., Chimenti, D.E. (eds.) Review of Progress in Quantitative Nondestructive Evaluation, pp. 2069-2076. Plenium Press, New York (1992)

93. Dace, G.E., Thompson, R.B., Brasche, L.J.H., Rehbein, D.K., Buck, O.: Nonlinear acoustics, a technique to determine microstructural changes in materials. In: Thompson, D.O., Chimenti, D.E. (eds.) Review of progress in quantitative nondestructive evaluation, pp. 1685-1692. Plenum Press, New York (1991)

94. Li, P., Yost, W.T., Cantrell, J.H., Salama, K.: Dependence of acoustic nonlinearity parameter on second phase. IEEE Ultrasonics Symposium. pp. 1113-1115 (1985)

95. Yost, W.T., Cantrell, J.H.: The effects of artificial aging of aluminum 2024 on its nonlinearity parameter. In: Thompson, D.O., Chimenti, D.E. (eds.) Review of Progress in Quantitative Nondestructive Evaluation, pp. 2067-2073. Plenum Press, New York (1993)

96. Sun, L., Kulkarni, S.S., Achenbach, J.D., Krishnaswamy, S.: Technique to minimize couplant-effect in acoustic nonlinearity measurements. J. Acoust. Soc. Am. 120, 2500-2505 (2006)

97. Baby, S., Kowmudi, B.N., Omprakash, C.M., Satyanarayana, D.V.V., Balasubramaniam, K., Kumar, V.: Creep damage assessment in titanium alloy using a nonlinear ultrasonic technique. Scr. Mater. 59, 818-821 (2008)

98. Nucera, C., Lanza di Scalea, F.: Nonlinear wave propagation in constrained solids subjected to thermal loads. J. Sound Vib. 333, 541-554 (2014)

99. Hurley, D.C., Balzar, D., Purtscher, P.T., Hollman, K.W.: Nonlinear ultrasonic parameter in quenched martensitic steels. J. Appl. Phys. 83, 4584-4588 (1998)

100. Li, W., Cho, Y., Lee, J., Achenbach, J.D.: Assessment of heat treated inconel X-750 alloy by nonlinear ultrasonics. Exp. Mech. 53, 775-781 (2012)

101. Kim, C.S., Park, I.K., Jhang, K.-Y.: Nonlinear ultrasonic characterization of thermal degradation in ferritic $2.25 \mathrm{Cr}-1 \mathrm{Mo}$ steel. NDT\&E Int. 42, 204-209 (2009)

102. Liu, S., Croxford, A.J., Neild, S.A., Zhou, Z.: Effects of experimental variables on the nonlinear harmonic generation technique. IEEE Trans. Ultrason. Ferroelectr. Freq. Control. 58, 1442-1451 (2011)

103. Na, J.K., Breazeale, M.A.: Ultrasonic nonlinear properties of lead zicronate-titanate ceramics. J. Acoust. Soc. Am. 95, 3213-3221 (1994)

104. Barnard, D.J.: Variation of nonlinearity parameter at low fundamental amplitudes. Appl. Phys. Lett. 74, 2447 (1999)

105. Cantrell, J.H., Yost, W.T.: Determination of Peierls stress from acoustic harmonic generation. Philos. Mag. Lett. 92, 128-132 (2012)

106. Cantrell, J.H.: Nonlinear dislocation dynamics at ultrasonic frequencies. J. Appl. Phys. 105, 1-7 (2009)

107. Yost, W.T., Cantrell, J.H.: Absolute ultrasonic displacement amplitude measurements with a submersible electrostatic acoustic transducer. Rev. Sci. Instrum. 63, 4182 (1992)

108. Yost, W.T., Breazeale, M.A.: Ultrasonic nonlinearity parameters and third-order elastic constants of germanium between 300 and 77K. Phys. Rev. B. 9, 510-516 (1974)

109. Hess, P., Lomonosov, A.M., Mayer, A.P.: Laser-based linear and nonlinear guided elastic waves at surfaces (2D) and wedges (1D). Ultrasonics. 54, 39-55 (2014)

110. Green, R.E.: Non-contact ultrasonic techniques. Ultrasonics 42, 9-16 (2004)

111. Stratoudaki, T., Hernandez, J.A., Clark, M., Somekh, M.G.: Cheap optical transducers (CHOTs) for narrowband ultrasonic applications. Meas. Sci. Technol. 18, 843-851 (2007) 
112. Collison, I.J., Stratoudaki, T., Clark, M., Somekh, M.G.: Measurement of elastic nonlinearity using remote laser ultrasonics and CHeap Optical Transducers and dual frequency surface acoustic waves. Ultrasonics 48, 471-477 (2008)

113. Stratoudaki, T., Ellwood, R., Sharples, S., Clark, M., Somekh, M.G., Collison, I.J.: Measurement of material nonlinearity using surface acoustic wave parametric interaction and laser ultrasonics. J. Acoust. Soc. Am. 129, 1721-8 (2011)

114. Ellwood, R., Stratoudaki, T., Sharples, S.D., Clark, M., Somekh, M.G.: Determination of the acoustoelastic coefficient for surface acoustic waves using dynamic acoustoelastography: An alternative to static strain. J. Acoust. Soc. Am. 135, 1064-1070 (2014)

115. Shui, G., Kim, J.-Y., Qu, J., Wang, Y.-S., Jacobs, L.J.: A new technique for measuring the acoustic nonlinearity of materials using Rayleigh waves. NDT E Int. 41, 326-329 (2008)

116. Shui, G., Wang, Y.: Ultrasonic evaluation of early damage of a coating by using second- harmonic generation technique. J. Appl. Phys. 111, 124902 (2012)

117. Rao, V.V.S.J., Kannan, E., Prakash, R.V., Balasubramaniam, K.: Fatigue damage characterization using surface acoustic wave nonlinearity in aluminum alloy AA7175-T7351. J. Appl. Phys. 104, 123508 (2008)

118. Morris, W.L., Buck, O., Inman, R.V.: Acoustic harmonic generation due to fatigue damage in high-strength aluminum. J. Appl. Phys. 50, 6737-6741 (1979)

119. Ogi, H., Hirao, M., Aoki, S.: Noncontact monitoring of surfacewave nonlinearity for predicting the remaining life of fatigued steels. J. Appl. Phys. 90, 438-442 (2001)

120. Cobb, A., Capps, M., Duffer, C., Feiger, J., Robinson, K., Hollingshaus, B.: Nonlinear ultrasonic measurements with EMATs for detecting pre-cracking fatigue damage. In: Thomson, D.O. and Chimenti, D.E. (eds.) Review of Progress in Quantitative Nondestructive Evaluation. pp. 299-306. AIP Publishing (2012)

121. Castaings, M., Cawley, P.: The generation, propagation, and detection of Lamb waves in plates using air-coupled ultrasonic transducers. J. Acoust. Soc. Am. 100, 3070-3077 (1996)

122. Wright, W.M.D., Hutchins, D.A., Hayward, G., Gachagan, A.: Ultrasonic imaging using laser generation and piezoelectric aircoupled detection. Ultrasonics 34, 405-409 (1996)

123. Zhu, J., Popovics, J.: Non-contact detection of surface waves in concrete using an air-coupled sensor. In: Thompson, D.O., Chimenti, D.E. (eds.) Review of Progress in Quantitative Nondestructive Evaluation, pp. 1261-1268. American Institute of Physics, Melville, NY (2002)

124. Tuzzeo, D., di Scalea, F.L.: Noncontact air-coupled guided wave ultrasonics for detection of thinning defects in aluminum plates. Res. Nondestruct. Eval. 13, 61-77 (2001)

125. Grandia, W.A., Fortunko, C.M.: NDE applications of air-coupled ultrasonic transducers. IEEE Ultrason. Symp. 1, 697-709 (1995)

126. Deighton, M.O., Gillespie, A.B., Pike, R.B., Watkins, R.D.: Mode conversion of Rayleigh and Lamb waves to compression waves at a metal-liquid interface. Ultrasonics 19, 249-258 (1981)

127. Bender, F.A., Kim, J.-Y., Jacobs, L.J., Qu, J.: The generation of second harmonic waves in an isotropic solid with quadratic nonlinearity under the presence of a stress-free boundary. Wave Motion. 50, 146-161 (2013)

128. Buck, O., Morris, W.L., Richardson, J.M.: Acoustic harmonic generation at unbonded interfaces and fatigue cracks. Appl. Phys. Lett. 33, 371-373 (1978)

129. Yost, W.T., Cantrell, J.H.: The effects of fatigue on acoustic nonlinearity in aluminum alloys. IEEE Ultrason. Symp. 947-955 (1992)

130. Cantrell, J.H.: Dependence of microelastic-plastic nonlinearity of martensitic stainless steel on fatigue damage accumulation. J. Appl. Phys. 100, 063508 (2006)
131. Kulkarni, S.S., Sun, L., Moran, B., Krishnaswamy, S., Achenbach, J.D.: A probabilistic method to predict fatigue crack initiation. Int. J. Fract. 137, 9-17 (2006)

132. Kulkarni, S.S., Achenbach, J.D.: Structural health monitoring and damage prognosis in fatigue. Struct. Heal. Monit. 7, 37-49 (2008)

133. Jhang, K.-Y., Kim, K.-C.: Evaluation of material degradation using nonlinear acoustic effect. Ultrasonics 37, 39-44 (1999)

134. Na, J.K., Yost, W.T., Cantrell, J.H.: Linear and nonlinear ultrasonic properties of fatigued $410 \mathrm{CB}$ stainless steel. In: Thompson, D.O., Chimenti, D.E. (eds.) Review of Progress in Quantitative Nondestructive Evaluation, p. 1347. Plenum Press, New York (1996)

135. Kumar, A., Torbet, C.J., Jones, J.W., Pollock, T.M.: Nonlinear ultrasonics for in situ damage detection during high frequency fatigue. J. Appl. Phys. 106, 024904 (2009)

136. Kumar, A., Adharapurapu, R.R., Jones, J.W., Pollock, T.M.: In situ damage assessment in a cast magnesium alloy during very high cycle fatigue. Scr. Mater. 64, 65-68 (2011)

137. Kumar, A., Torbet, C.J., Pollock, T.M.: Wayne Jones, J.: In situ characterization of fatigue damage evolution in a cast $\mathrm{Al}$ alloy via nonlinear ultrasonic measurements. Acta Mater. 58, 2143-2154 (2010)

138. Campos-Pozuelo, C., Vanhille, C., Gallego-Juárez, J.A.: Comparative study of the nonlinear behavior of fatigued and intact samples of metallic alloys. IEEE Trans. Ultrason. Ferroelectr. Freq. Control. 53, 175-84 (2006)

139. Shui, G., Wang, Y.-S., Gong, F.: Evaluation of plastic damage for metallic materials under tensile load using nonlinear longitudinal waves. NDT E Int. 55, 1-8 (2013)

140. Kim, J.-Y., Qu, J., Jacobs, L.J., Littles, J.W., Savage, M.F.: Acoustic Nonlinearity Parameter Due to Microplasticity. J. Nondestruct. Eval. 25, 28-36 (2006)

141. Rao, V.V.S.J., Kannan, E., Prakash, R.V., Balasubramaniam, K.: Observation of two stage dislocation dynamics from nonlinear ultrasonic response during the plastic deformation of AA7175T7351 aluminum alloy. Mater. Sci. Eng. A. 512, 92-99 (2009)

142. Cantrell, J.H., Yost, W.T.: Effect of precipitate coherency strains on acoustic harmonic generation. J. Appl. Phys. 81, 2957-2962 (1997)

143. Mukhopadhyay, A., Sarkar, R., Punnose, S., Valluri, J., Nandy, T.K., Balasubramaniam, K.: Scatter in nonlinear ultrasonic measurements due to crystallographic orientation change induced anisotropy in harmonics generation. J. Appl. Phys. 111, 054905 (2012)

144. Xiang, Y., Deng, M., Xuan, F.-Z., Liu, C.-J.: Experimental study of thermal degradation in ferritic Cr-Ni alloy steel plates using nonlinear Lamb waves. NDT\&E Int. 44, 768-774 (2011)

145. Metya, A., Ghosh, M., Parida, N., Palit Sagar, S.: Higher harmonic analysis of ultrasonic signal for ageing behaviour study of C-250 grade maraging steel. NDT E Int. 41, 484-489 (2008)

146. Jeong, H., Nahm, S.-H., Jhang, K.-Y., Nam, Y.-H.: A nondestructive method for estimation of the fracture toughness of $\mathrm{CrMoV}$ rotor steels based on ultrasonic nonlinearity. Ultrasonics 41, 543549 (2003)

147. Park, J., Kim, M., Chi, B., Jang, C.: Correlation of metallurgical analysis and higher harmonic ultrasound response for long term isothermally aged and crept FM steel for USC TPP turbine rotors. NDT E Int. 54, 159-165 (2013)

148. Abraham, S.T., Albert, S.K., Das, C.R., Parvathavarthini, N., Venkatraman, B., Mini, R.S., Balasubramaniam, K.: Assessment of sensitization in AISI 304 stainless steel by nonlinear ultrasonic method. Acta Metall. Sin. 26, 545-552 (2013)

149. Xiang, Y., Deng, M., Xuan, F.-Z., Liu, C.-J.: Cumulative secondharmonic analysis of ultrasonic Lamb waves for ageing behavior study of modified-HP austenite steel. Ultrasonics 51, 974-981 (2011) 
150. Balasubramaniam, K., Valluri, J.S., Prakash, R.V.: Creep damage characterization using a low amplitude nonlinear ultrasonic technique. Mater. Charact. 62, 275-286 (2011)

151. Valluri, J.S., Balasubramaniam, K., Prakash, R.V.: Creep damage characterization using non-linear ultrasonic techniques. Acta Mater. 58, 2079-2090 (2010)

152. Narayana, V.J.S., Balasubramaniam, K., Prakash, R.V., Thompson, D.O., Chimenti, D.E.: Detection and prediction of creepdamage of copper using nonlinear acoustic techniques. In: Thompson, D.O., Chimenti, D.E. (eds.) Review of Progress in Quantitative Nondestructive Evaluation, pp. 1410-1417. American Institute of Physics, San Diego (2010)

153. Kim, C.S., Lissenden, C.J.: Precipitate contribution to the acoustic nonlinearity in nickel-based superalloy. Chin. Phys. Lett. 26, 086107 (2009)

154. Matlack, K.H., Wall, J.J., Kim, J.-Y., Qu, J., Jacobs, L.J.: Nonlinear ultrasound to monitor radiation damage in structural steel. 6th European Workshop on Structural Health Monitoring. pp. 1-8 (2012)

155. Odette, G.R., Lucas, G.E.: Embrittlement of nuclear reactor pressure vessels. JOM 53, 18-22 (2001)
156. Zeitvogel, D.T., Matlack, K.H., Kim, J.-Y., Jacobs, L.J., Singh, P.M., Qu, J.: Characterization of stress corrosion cracking in carbon steel using nonlinear Rayleigh surface waves. NDT E Int. 62, $144-152$ (2014)

157. Biwa, S., Nakajima, S., Ohno, N.: On the acoustic nonlinearity of solid-solid contact with pressure-dependent interface stiffness. J. Appl. Mech. 71, 508-515 (2004)

158. Hirsekorn, S.: Nonlinear transfer of ultrasound by adhesive joints-a theoretical description. Ultrasonics 39, 57-68 (2001)

159. Wegner, A., Koka, A., Janser, K., Netzelmann, U., Hirsekorn, S., Arnold, W.: Assessment of the adhesion quality of fusion-welded silicon wafers with nonlinear ultrasound. Ultrasonics 38, 316-321 (2000)

160. Solodov, I.Y.: Ultrasonics of non-linear contacts: propagation, reflection and NDE-applications. Ultrasonics 36, 383-390 (1998)

161. Van Den Abeele, K., Desadeleer, W., De Schutter, G., Wevers, M.: Active and passive monitoring of the early hydration process in concrete using linear and nonlinear acoustics. Cem. Concr. Res. 39, 426-432 (2009) 